\title{
The rich young cluster NGC 6530: a combined X-ray-optical-infrared study ${ }^{\star}$
}

\author{
F. Damiani, L. Prisinzano, G. Micela, and S. Sciortino
}

\begin{abstract}
INAF - Osservatorio Astronomico di Palermo G.S.Vaiana, Piazza del Parlamento 1, 90134 Palermo, Italy e-mail: damiani@astropa.unipa.it
\end{abstract}

Received 19 July 2005 / Accepted 1 May 2006

ABSTRACT

\begin{abstract}
We present a combined X-ray, optical, and IR (2MASS) study of NGC 6530, complementing our previous studies of this cluster. We consider different indicators of IR excesses, which can be taken as an indicator of circumstellar disks and therefore of pre-mainsequence status. We used reddening-free indices to ensure that our results are unaffected by highly irregular, differential reddening. More than the study of the JHK bands alone (33 IR-excess stars found), we found it useful to compare various optical and IR colors, finding overall 333 stars with optical-IR excess. In the field of the previously studied Chandra ACIS-I observation, we found 196 stars with optical-IR excess, of which 120 have not been previously found in X-rays. The total number of estimated cluster members thus becomes $\geq 1100$. The estimated disk frequency in the ACIS field is $\sim 20 \%$. By considering different optical-IR excess indices, we find only partially overlapping subsamples, corresponding to different characteristics of their spectral energy distributions (longeror shorter-wavelength IR excesses, or blue-violet excess). In a region displaced towards northwest with respect to the known cluster center, we find an unexpected concentration of stars with optical-IR excesses, most of which are not detected in X-rays. The spectral energy distribution of some of these objects shows more extreme excesses with respect to most ordinary T Tauri stars, which suggests circumstellar disks with very small inner holes and high accretion rates; some objects are best interpreted as being surrounded by reflection nebulosities, as found in some optically detected Class I objects in nearer star-forming regions. These reflection-nebulae candidates have the lowest X-ray detection rate among all subsamples considered here. Optical-IR excess stars in the north of NGC 6530 are nearly co-spatial with a sub-population of cluster stars older than the central cluster stars. This leads to the conclusion that in these northern regions of NGC 6530, far from massive cluster stars, star formation (and disk evolution) has proceeded rather undisturbed over longer time periods than near the cluster center, where most massive stars are found, and most stars lack substantial disks and strong accretion.
\end{abstract}

Key words. Galaxy: open clusters and associations: individual: NGC 6530 - stars: coronae - stars: circumstellar matter X-rays: stars - stars: pre-main sequence

\section{Introduction}

The high-mass star formation region NGC 6530 has already been studied since the late fifties (Walker 1957; The 1960), but has been receiving much attention recently (e.g. van den Ancker 1997; Sung et al. 2000 (hereafter SCB), Rauw et al. 2002; Damiani et al. 2004). In particular, its low-mass population was very poorly known before the X-ray study performed with Chandra by Damiani et al. (2004, hereafter Paper I), who detected 884 X-ray sources, of which $90-95 \%$ are estimated to be low-mass cluster members. The known low-mass population of NGC 6530 has thus increased from a few tens of stars (van den Ancker 1997, SCB) to several hundred stars.

Since most of the X-ray sources found in Paper I remained without optical counterparts, deep (down to $V \sim 23$ ) $B V I$ photometry (taken with the ESO WFI@2.2m) was retrieved from the ESO archive and analyzed by Prisinzano et al. (2005, hereafter Paper II). This permitted the identification of 774 X-ray sources with optical stars, whose positions on the HR diagram confirmed the nature of these stars as low-mass pre-main-sequence (PMS) objects. Moreover, thanks to the very large number statistics of WFI detected stars, we were able to derive a new value for the

^ Appendices A-C are only available in electronic form at http://www . aanda.org distance of NGC 6530 (1250 pc, Paper II), near the bottom of the range found by previous studies (e.g. The 1960). In Paper II we also derived the cluster IMF.

Another interesting result, first found in Paper I and then confirmed with better stellar statistics in Paper II, is the presence of an age gradient from the northwestern part of NGC 6530 towards the south and southeastern parts, the latter containing the youngest stars, which happen to also be those with the highest frequency of X-ray flares across the cluster. These results strengthen the existing arguments suggesting a "sequential" star formation in this region (Lada et al. 1976).

In Paper I, identifications of X-ray sources with infrared (IR) sources from the 2MASS survey (Cutri et al. 2003) were also presented, but only briefly discussed. The purpose of this paper is therefore to examine the relationships between the X-ray data on NGC 6530 stars from Paper I, the optical photometry from Paper II, and the 2MASS IR data, with the double perspective of identifying the cluster population as completely as possible, and of studying the properties of those cluster stars found there. Since the observational data used here have already been presented (including the X-ray to optical and X-ray to IR identifications), we can start directly with their analysis.

In Sect. 2, we study the overall distributions of these stars in space, in color-color, and in color-magnitude diagrams. In Sect. 3 we define suitable reddening-free indices and use them to 


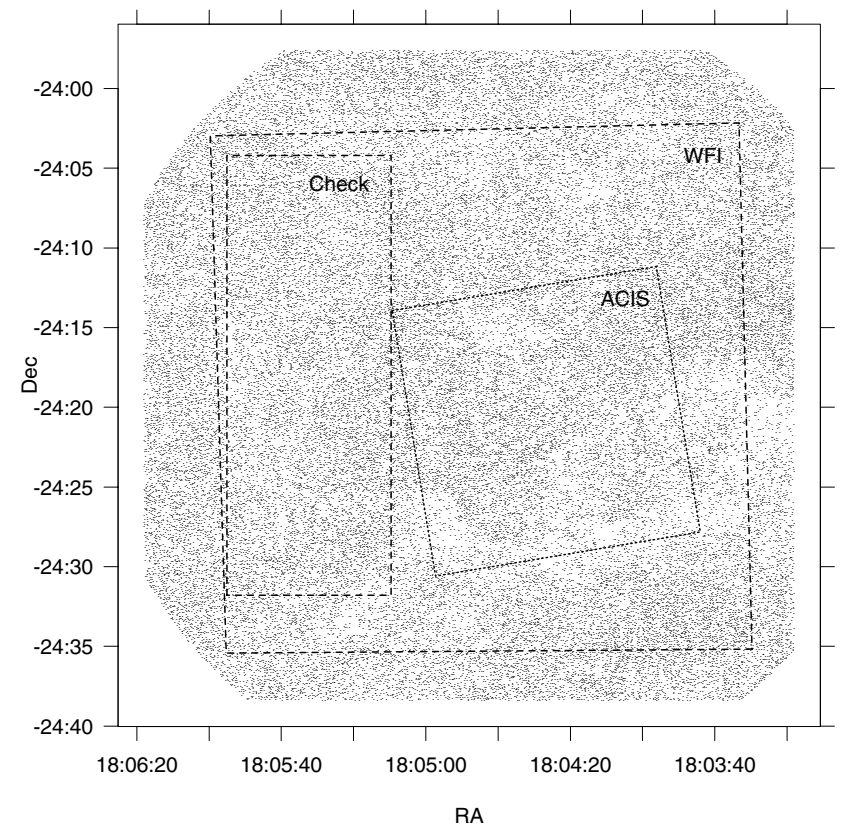

Fig. 1. Spatial distribution of all good 2MASS sources in the NGC 6530 region. Indicated are the WFI and ACIS FOVs, as well as the selected Check Field.

select stars with color excesses. Some statistics of the new stellar population is presented in Sect. 4. The nature of stars with color excesses is studied further in Sect. 5. Section 6 is a discussion of the results obtained, and Sect. 7 summarizes all conclusions of this work. A detailed study of all sources of uncertainties is made in Appendix A. A second appendix describes a method for computing the number of spurious identifications between two catalogs, and another is devoted to computing an optical extinction map of NGC 6530.

\section{Distribution of IR sources}

In this section we first examine the distribution of 2MASS IR sources in space, we then define suitable subregions, and study the distributions of objects in IR color-color and color-magnitude diagrams for each such subregion, for deriving average properties of cluster and field-star populations.

\subsection{Space distribution of $2 M A S S$ sources}

NGC 6530 is projected against the bright emission nebula M 8 ("Lagoon Nebula"), the illuminated part of a giant molecular cloud from which the cluster itself formed (see e.g. Fig. 1 of Paper I). The cloud causes large and spatially variable optical extinction, evident from the study of the HR diagram of stars in the region (Paper II), but also suggested by a spatially variable density of field stars, most of which farther out than the cloud. For reference, we plot in Fig. 1 the space distribution of all "good" 2MASS sources in the region of interest (see Paper I for the selection of good IR sources), and the boundaries of the Chandra ACIS-I field of view (FOV) studied in Paper I and of the WFI mosaic image studied in Paper II, which is much larger than the ACIS FOV. Despite the much smaller extinction in the IR compared to optical wavelengths, a pronounced deficit of field stars is obvious, e.g. in the lower parts of the ACIS FOV, to its west (right), and near the northwestern part of the WFI FOV. With the purpose of obtaining a reference comparison field, we defined the rectangular region labeled "Check" in Fig. 1, which has the same area as the ACIS FOV (but without available X-ray data) and is free of star-density irregularities. Its choice as a comparison field is corroborated by the fact that the majority of NGC 6530 members are estimated to lie inside the ACIS FOV, judging from the density profile of $\mathrm{X}$-ray detected sources (Paper I). We do not mean that this can be taken as a representative control field for the Galactic plane: it is still projected onto the molecular cloud and may contain some cluster stars, but we will find it useful when comparing it with the ACIS field just nearby.

\subsection{IR color-magnitude and color-color diagrams}

In Paper I (Fig. 5) we showed a $(J-K, H-K)$ color-color diagram of NGC 6530 (in the ACIS FOV) and noted that: $a$ ) most $\mathrm{X}$-ray detected stars have nearly normal photospheric colors with nominal or slightly higher extinction and are the best candidate cluster members; $b$ ) a large number of other objects, mostly undetected in X-rays, are spread along the reddening vector, probably background field (giant) stars; $c$ ) a few stars, in many cases detected in X-rays, show redder colors than justified by extinction, suggesting IR excesses; $d$ ) at least some X-ray detected cluster stars with apparently normal IR colors are too red (in $J-K$ or $H-K)$ for their $V-I$ color, again suggesting IR excesses.

The $(J, J-K)$ color-magnitude diagram of our NGC 6530 ACIS FOV (not shown here) leads to similar conclusions, with most X-ray sources clustering between the 1-2 Myr isochrones (from Siess et al. 2000 ${ }^{1}$ ), except for a few tens of X-ray sources, found at redder IR colors; a large population of highly reddened (up to $A_{\mathrm{V}} \sim 20$ ) X-ray undetected objects is also evident. The $(J, J-K)$ diagram relative to the Check field (defined in Fig. 1) is distinctly different from that of the ACIS FOV: the "cluster" region defined by X-ray-selected members and massive stars is much less populated, and extremely red colors are rarer, with stars accumulating instead at intermediate reddenings around $A_{\mathrm{V}} \sim 7$ (see Appendix C). This confirms the suitability of our chosen Check field as a reference that it almost devoid of cluster members and strong extinction nonuniformities.

Since high and unknown reddening and intrinsic IR emission excesses are competing effects, displacing stars in color-magnitude or color-color diagrams, they are not easy to disentangle from each other, but we attempt to do it in the following sections. A star definitely showing IR excess emission in a star-forming region such as NGC 6530 is regarded as a cluster member that is still surrounded by a dust disk or envelope.

\section{A search for IR-excess stars}

In order to select stars showing photometric excesses in various spectral ranges, we define here suitable reddening-free indices, based on combinations of IR and optical colors. In this way, the census of known NGC 6530 members (in addition to the X-ray selected members from Paper I) is enriched by several hundred stars, as summarized in Table 1.

\footnotetext{
1 There are substantial differences between PMS evolutionary tracks computed by different authors, as discussed e.g. by Baraffe et al. (2002). However, our adoption of the Siess et al. (2000) models does not noticeably affect our results, which are mostly based on comparisons of the colors of NGC 6530 stars with those of normal stars, the latter being taken from the Kenyon \& Hartmann (1995) compilation.
} 
Table 1. Number of candidate members of NGC 6530 for each selection method. Panel 1a: stars in the ACIS field; diagonal terms are the total numbers of stars for each method, while off-diagonal terms are those found by two methods simultaneously. Panel $1 \mathrm{~b}$ : the same representation for the entire WFI field, including the ACIS field (X-ray sources are not reported as there is no additional X-ray information outside the ACIS field). Panels $2 \mathrm{a}$ and $\mathrm{b}$ : members found exclusively by one method, in the ACIS and WFI fields. Panels $3 \mathrm{a}$ and b: members found by any of the $Q$ indices, regardless of X-ray detection, including X-ray sources and excluding them, respectively, in the ACIS and WFI fields.

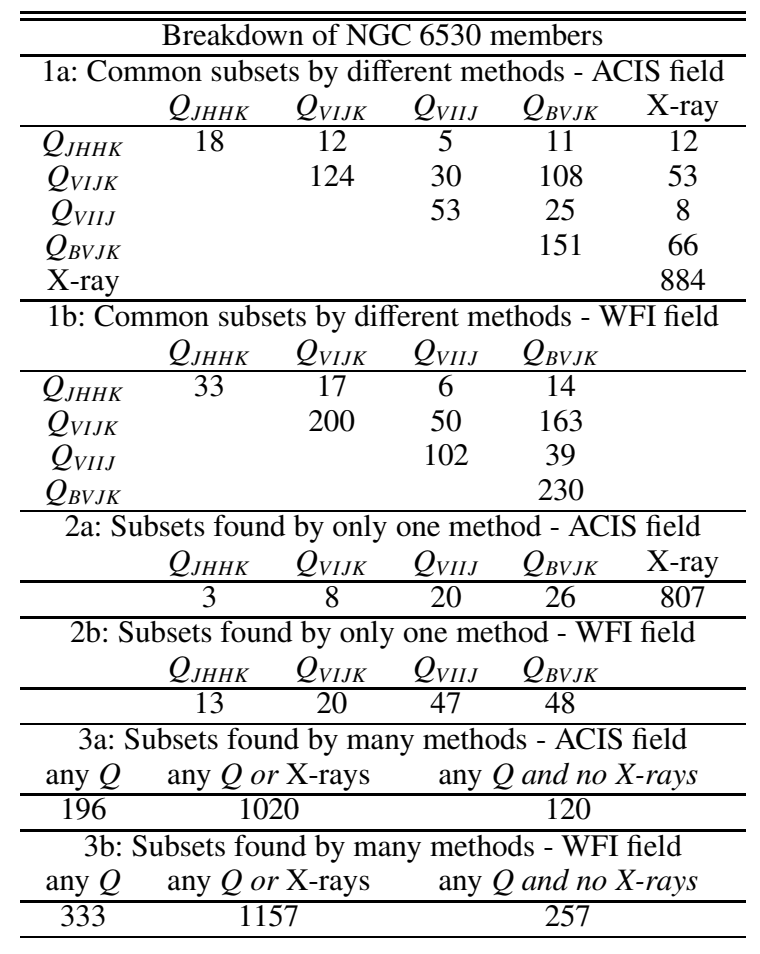

\subsection{The extinction-free IR index $Q_{J H H K}$}

In a $(J-H, H-K)$ color-color diagram, only a narrow strip can be populated by normal main-sequence or giant stars by varying the optical extinction, while stars outside of this strip have peculiar emission, independent of extinction. This fact has been used by many authors to select classical T Tauri stars or young stellar objects, falling preferentially in a "T Tauri star locus" (Meyer et al. 1997). On this basis, we define a reddening-free index, analogous to the Johnson $Q$ index, providing a measure of the deviation from the "permitted" strip of reddened normal stars, as

$Q_{J H H K}=(J-H)-E(J-H) / E(H-K) \times(H-K)$.

The ratio $E(J-H) / E(H-K)$ in NGC 6530 is determined very well directly from observational data, as the slope of the background star distribution in the IR color-color diagram. We find good agreement with the extinction law of Rieke \& Lebofsky (1985), $E(J-H) / E(H-K)=1.7$, which we adopt here, while poorer agreement is found with the value (1.55) given by Mathis (1990).

Figure 2 shows the $Q_{J H H K}$ index versus $H-K$ for all 2MASS sources in the ACIS FOV. A vector is also shown in the figure for an excess affecting only the $K$ band, a very crude approximation of the true IR excess spectrum. Crosses in Fig. 2 indicate $\mathrm{X}$-ray sources, most of which appear to have normal colors with somewhat more than nominal reddening, but some spread along the " $K$-band excess vector" towards values of $Q_{J H H K}$ too low for

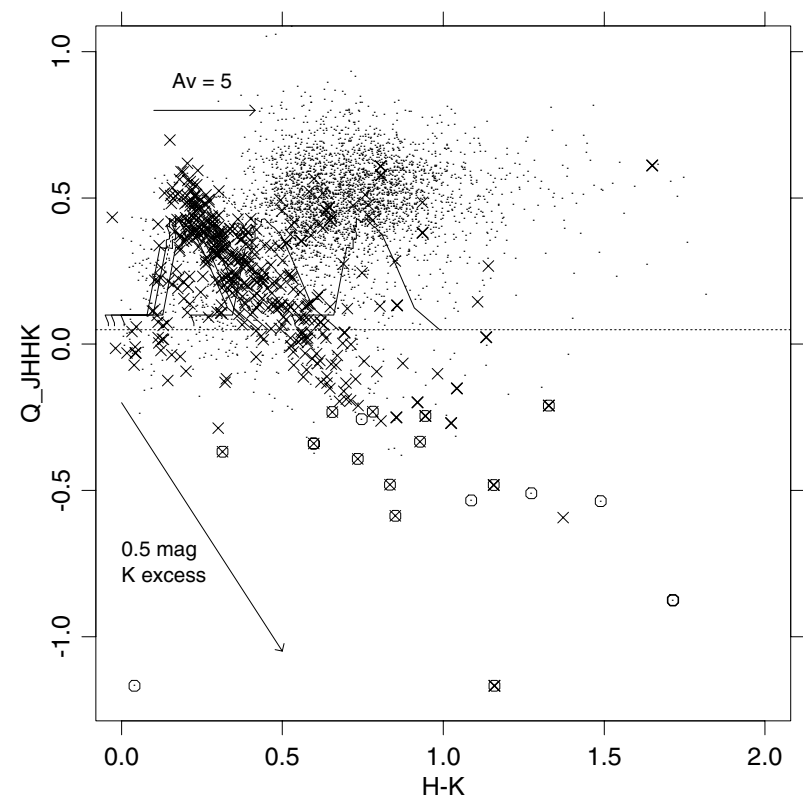

Fig. 2. Plot of the reddening-free index $Q_{J H H K}$ (see text) vs. $H-K$, for objects in the ACIS FOV. X's are X-ray detected sources, while small dots are sources not detected in X-rays. The reddening and excess vectors are shown. ZAMS stellar colors are shown for $A_{\mathrm{V}}$ equal to the minimum, average, and maximum values for NGC 6530 (from SCB) and $A_{\mathrm{V}}=5$ and 10 , respectively. A dashed line is the locus of giant stars. Thick $\times$ 's are hard X-ray sources. The horizontal dotted line indicates the lowest $Q_{J H H K}$ value attained by normal photospheres. Empty circles are sources with significant excess in the $Q_{J H H K}$ index. Thick circles are candidate protostars $\left(Q_{J H H K}\right.$-excess sources without optical counterpart).

normal stars. We have selected those IR sources distant by more than $3 \sigma$ from the permitted "normal" locus $\left(Q_{J H H K}>0.05\right.$, using colors from Kenyon \& Hartmann 1995), as definite IR excess objects (circles in Fig. 2). There are 18 such objects in the ACIS FOV, and 33 in the entire WFI FOV. We call these objects " $Q_{J H H K}$ excess" stars (see Table 1 for a summary). Only $6 Q_{J H H K}$-excess objects in the ACIS FOV have not already been detected in X-rays, so are actually enlarging our sample of candidate cluster members. Seven $Q_{J H H K}$-excess objects in the entire WFI FOV have not been detected in our optical data (nor in the SCB data), and are therefore candidate protostars in NGC 6530 (Fig. 2). Only one of these candidate protostars falls in the ACIS FOV, but is not detected in X-rays.

The fact that most of the X-ray sources falling in the elongated band below $Q_{J H H K} \sim 0.05$ do not show individually significant IR excesses does not entitle us to consider them entirely normal; it is instead more likely that this IR-based method (with the relatively large 2MASS photometric errors at these magnitudes) is not sensitive enough. The analogous diagram for the Check field (not shown) shows that background stars are clustered more towards lower extinction values $\left(A_{\mathrm{V}} \sim 7\right)$, and there are only $4 Q_{J H H K}$-excess objects, a significantly smaller number than in the ACIS FOV.

\subsection{Extinction-free indices for mixed optical-IR diagrams: V-I vs. J-K}

We noted in Paper I that many X-ray sources in NGC 6530 appear to have IR colors redder than expected from their optical (e.g. $V-I$ ) colors. By comparing the (photospheric) $V-I$ color 


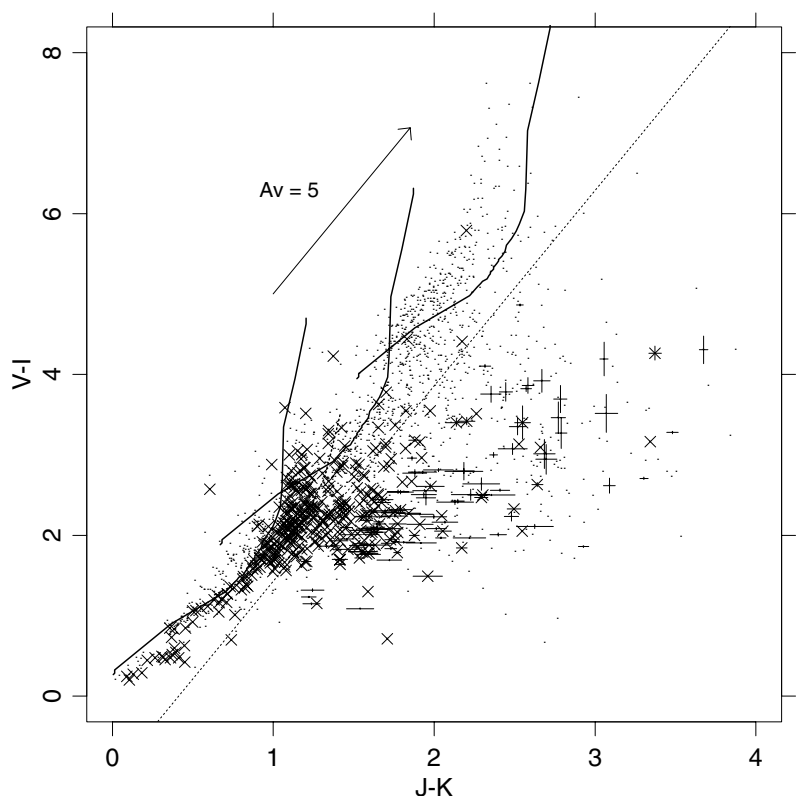

Fig. 3. A $(V-I, J-K)$ optical-infrared color-color diagram, for stars in the ACIS FOV. $\times$ 's are X-ray detected stars, while small dots are $\mathrm{X}$-ray undetected ones. The ZAMS locus (solid line) is shown for the nominal cluster extinction and for $A_{\mathrm{V}}=5$ and 10, respectively. The reddening vector is also shown. The dotted line indicates a limit for normal photospheric colors. Stars significantly to the right of this limit, plotted with error bars, are $Q_{V I J K}$-excess stars.

with the (disk-sensitive) $J-K$ color, we obtain a reliable indication of the presence of circumstellar disks.

A $(V-I, J-K)$ two-color diagram for the ACIS FOV is shown in Fig. 3. As in the IR color-color diagram, there is a region in the diagram that cannot be populated by normal mainsequence or giant stars for any reddening. Main-sequence colors are taken from Kenyon \& Hartmann (1995). We have shown a representative lower boundary of normal star locations, slightly separated from the exact lower envelope of reddened ZAMS loci to take possible uncertainties in the normal-star colors into account. We also decided the position of this boundary from inspection of the analogous diagram for the Check field, which is much less "contaminated" by cluster stars than the ACIS FOV. The adopted definition is somewhat subjective, but on the conservative side, in order not to mistake normal stars as spurious cluster members.

We thus define another reddening-free index, mixing optical and IR colors, as

$Q_{V I J K}=(J-K)-E(J-K) / E(V-I) \times(V-I)$,

such that high values of the $Q_{V I J K}$ index correspond to stars with an IR excess with respect to their optical color. With respect to the reference boundary defined in Fig. 3, equivalent to a maximum allowed $Q_{V I J K}=0.41$, we define as $Q_{V I J K}$-excess stars all stars whose excess is discrepant by more than $3 \sigma$ and with errors in all of the VIJK magnitudes smaller than 0.1 mag, together with stars whose excess is discrepant by more than $5 \sigma$ and errors in the VIJK magnitudes up to $0.2 \mathrm{mag}$. Moreover, only stars with offsets between their WFI and 2MASS positions that are smaller than 0.4 arcsec are included (rather than 0.8 arcsec as for the general sample), to minimize the number of spurious optical-IR identifications (see Appendix A). Stars with a significant $Q_{V I J K}$ excess are indicated by their error bars in Fig. 3. Whenever a multiple optical-IR identification was found,

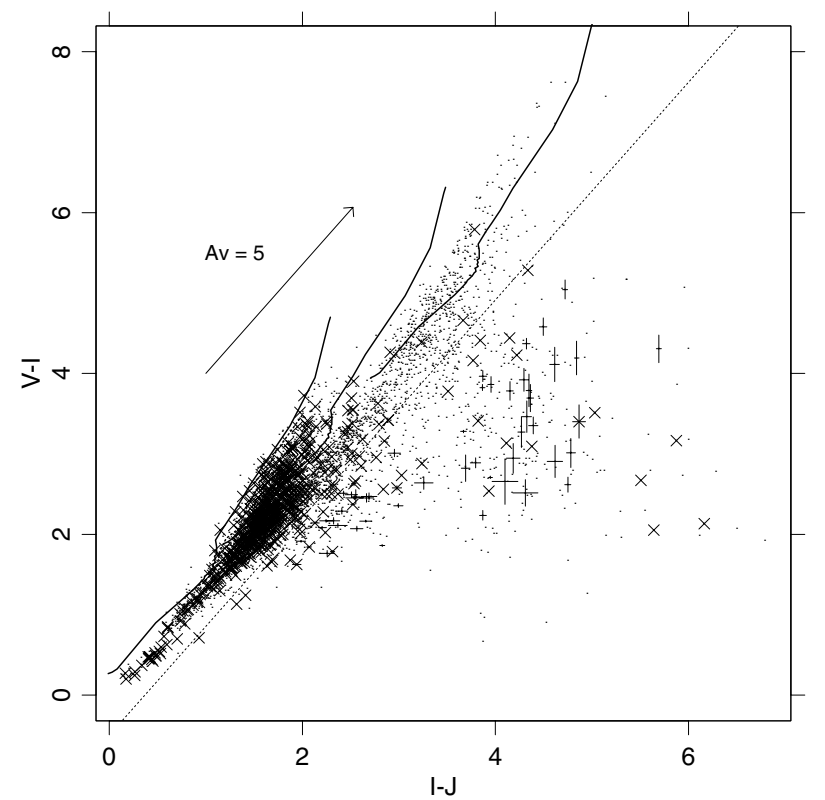

Fig. 4. A $(V-I, I-J)$ diagram, for stars in the ACIS FOV. Symbols are as in Fig. 3. Stars plotted with error bars have significant $Q_{V I I J}$ excesses.

a star was considered to have a significant excess only if all of its possible IR matches show an excess, and each such multiple match is counted only once in the statistics of excess sources below. Our definition is rather conservative, since there are many (hundreds) stars with errors in the VIJK magnitudes larger than $0.1 \mathrm{mag}$, and whose position in the diagram is still discrepant with respect to the limiting line by more than $3 \sigma$; however, as we discuss in Appendix A, these can be more severely contaminated by chance identifications, while our definition ensures a very low level of contamination.

In this way we find $124 Q_{V I J K}$ excess stars in the ACIS FOV, a much smaller number (9) in the Check field, and 200 in the entire WFI FOV (see Table 1). Stars in the ACIS FOV show, moreover, much larger excesses with respect to those in the Check field. Overall, this method is indeed able to select IR excess stars more efficiently than the approach based on the index $Q_{J H H K}$ discussed above, yielding 71 IR excess stars in the ACIS FOV that were not previously detected in X-rays (Paper I). This additional population of NGC 6530 amounts to $7.4 \%$ of the total known population falling in the ACIS FOV, selected from either X-rays or IR excess.

\subsection{Optical-IR indices: V-I vs. I-J}

Since circumstellar disks are not isothermal but have a distribution of temperatures (hotter near the star), shorter-wavelength colors (e.g., $I-J$ ) may be useful for sampling conditions in its innermost parts. Since there is evidence that circumstellar disks around low-mass pre-main-sequence stars do not extend inward down to the stellar surface, but are often truncated in their innermost parts (Bertout et al. 1988; Hillenbrand et al. 1992; Lada \& Adams 1992; Kenyon et al. 1996), the $I-J$ color is not expected to show an excess with respect to photospheric values, unlike the $J-K$ color (this justifies our earlier simplification of the IR excess as affecting only the $K$ band).

We did, however, find that this is often not the case in NGC 6530. Figure 4 is a color-color diagram comparing the $V-I$ and $I-J$ colors, for the ACIS FOV. Again, error bars indicate stars with significant discrepancies with respect to the 


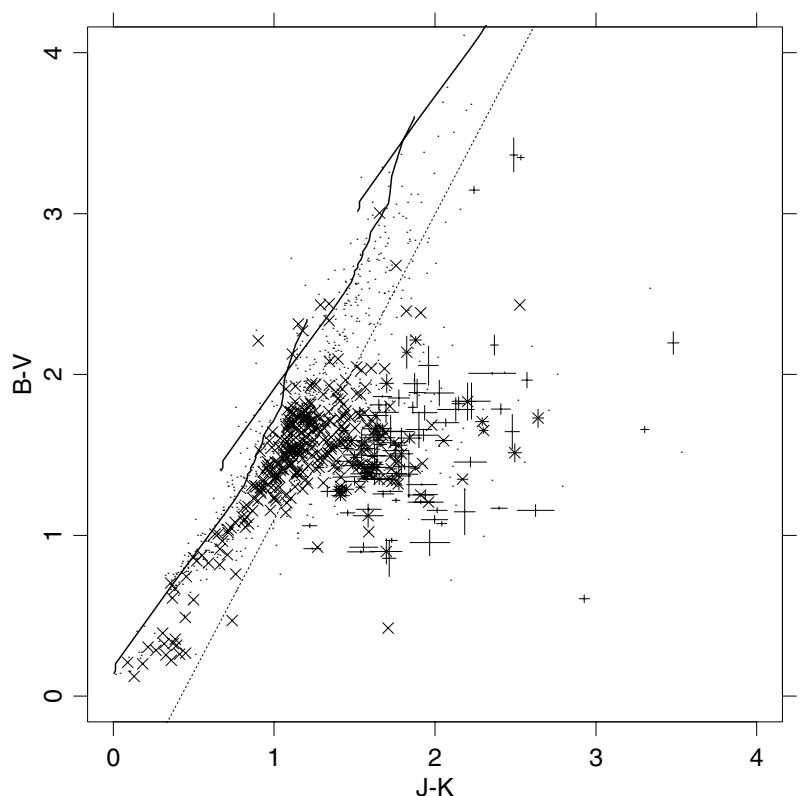

Fig. 5. $(B-V, J-K)$ diagram, analogous to Fig. 3, for stars in the ACIS FOV.

fiducial boundary, chosen in the same way as in Fig. 3. Also in this case, we define an index $Q_{V I I J}=(I-J)-E(I-J) / E(V-$ $I) \times(V-I)$. The maximum allowed value of this index for normal photospheric emission is $Q_{V I I J}=0.37$. We applied the same criterion used above to define $Q_{V I J K}$ excess stars, and now find stars with $Q_{V I I J}$ excess. As seen in Fig. 4, the basic pattern is similar to that of Fig. 3. Most X-ray detected stars occupy the ZAMS locus at low reddening, indicating that in X-ray detected cluster members there is indeed little non-photospheric excess emission at $J$. However, despite the very small $I-J$ excess found in most X-ray detected cluster members, 53 stars with significant $Q_{V I I J}$ excess are found in the ACIS FOV, mostly (45) not detected in X-rays. Only 30 of these excess stars have already been found as $Q_{V I J K}$-excess stars. A few $Q_{V I I J}$ excess stars (12) are also found in the Check field, a similar number as $Q_{V I J K}$-excess stars. The total number of $Q_{V I I J}$ excess stars found in the entire WFI FOV is 102 (Table 1). The existence of so many stars with a $Q_{V I I J}$ excess is puzzling, so we examine these objects in detail in the following sections. In Fig. 4 we see that these objects appear to split into two equally populated subgroups with "weak" and "strong" $Q_{V I I J}$ excess, respectively, the cutoff being around $I-J \sim 3.5$.

\subsection{Optical-IR indices: B-V vs. J-K}

We finally consider another pair of colors from very different wavelength ranges, $B-V$ and $J-K$, as shown in Fig. 5. We define the corresponding index $Q_{B V J K}=(J-K)-E(J-K) / E(B-$ $V) \times(B-V)$, describing the deviation from normal colors in the $(B-V, J-K)$ plane. The threshold for excess detection is now assumed to be $Q_{B V J K}=0.42$. Since our stars are on average very red, $B$ magnitudes are not available for all of them. Using the same selection procedure as above, we find 151 stars with $Q_{B V J K}$ excesses in the ACIS FOV, 12 in the Check field, and 230 in the entire WFI FOV (Table 1).

Among the 151 stars in the ACIS FOV, 85 have not been detected in X-rays, and 43 do not show any other kind of IR excess (from $Q_{J H H K}, Q_{V I J K}$, or $Q_{V I I J}$ indices); the number of the latter group rises to 65 across the entire WFI FOV. This justifies

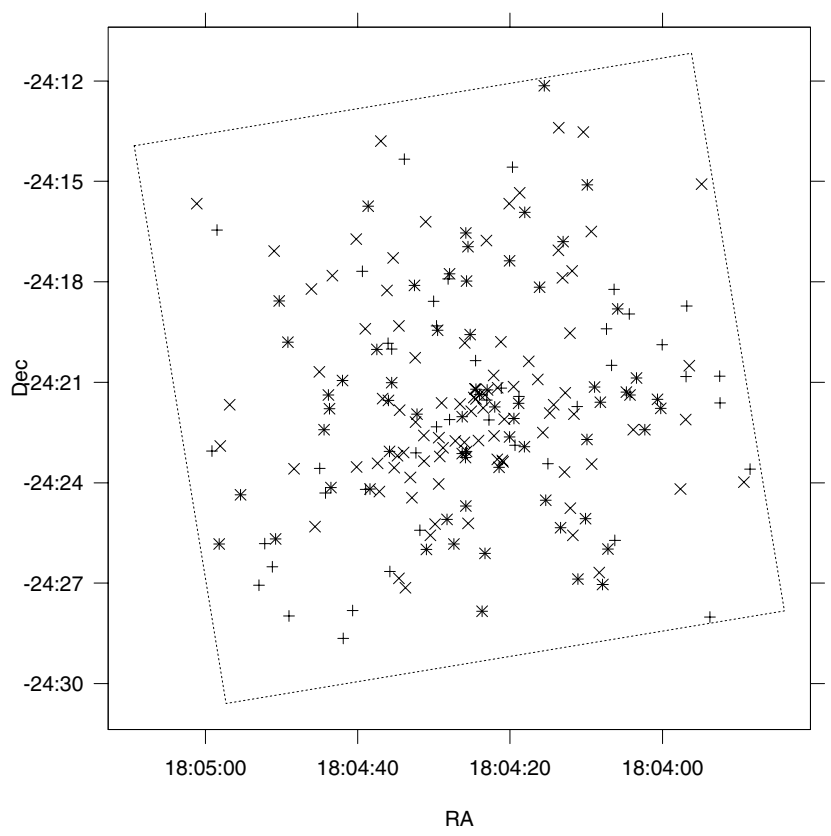

Fig. 6. Map of all X-ray sources without a 2MASS ( $\times$ 's) or WFI object (plus signs) identification, strongly clustered around the cluster center.

the use of this additional method and, at the same time, is something unexpected; all of these stars have measured $V-I$ colors, so that a $J-K$ excess should have appeared when studying the $Q_{V I J K}$ index. Some degree of uncertainty is introduced by our somewhat subjective placing of the "fiducial" boundary in the diagrams of Figs. 3 and 5. More probable, however, is that the excess detected in the $(B-V, J-K)$ plane is not simply a $J-K$ excess, as assumed in the discussion of $Q_{V I J K}$ (and as will be justified in the next section), but is also affected by a blueward displacement of the $B-V$ color, making the total displacement more significant. We found in Paper I that a number of cluster stars with $V<17$ exhibit a strong violet $(U-B)$ excess, and it is plausible that a significant (although less strong) excess may appear in the $B$ band too. Unfortunately, our photometry of Paper II does not comprise $U$ band measurements. These short-wavelengths excesses might be due to very strong chromospheric activity (accompanying strong X-ray activity in our X-ray detected sample), or to "hot spots" at the base of "accretion columns", where falling circumstellar matter impacts on the stellar surface (Calvet \& Gullbring 1998). The chromospheric hypothesis is strengthened by the fact that among the 43 new $B-V$ excess stars in ACIS FOV, a fairly large fraction (17) are detected in X-rays.

\section{Statistics of candidate NGC 6530 members}

We summarize the statistics of the new NGC 6530 members selected by these methods in Table 1 . In addition to the statistics mentioned in the previous section, the table lists additional statistics. The total number of (candidate) cluster members selected through either X-rays or one of the different optical-IR excesses is 1157 in the whole WFI FOV and 1020 in the ACIS FOV. These numbers include 153 X-ray sources without WFI or 2MASS identifications, whose clustered spatial distribution strongly suggests that they are mostly members of NGC 6530 (Fig. 6). The number of those selected by any IR-excess index, regardless of X-ray detection, is 333 for the whole WFI FOV and 196 in the ACIS FOV. Stars selected by any 


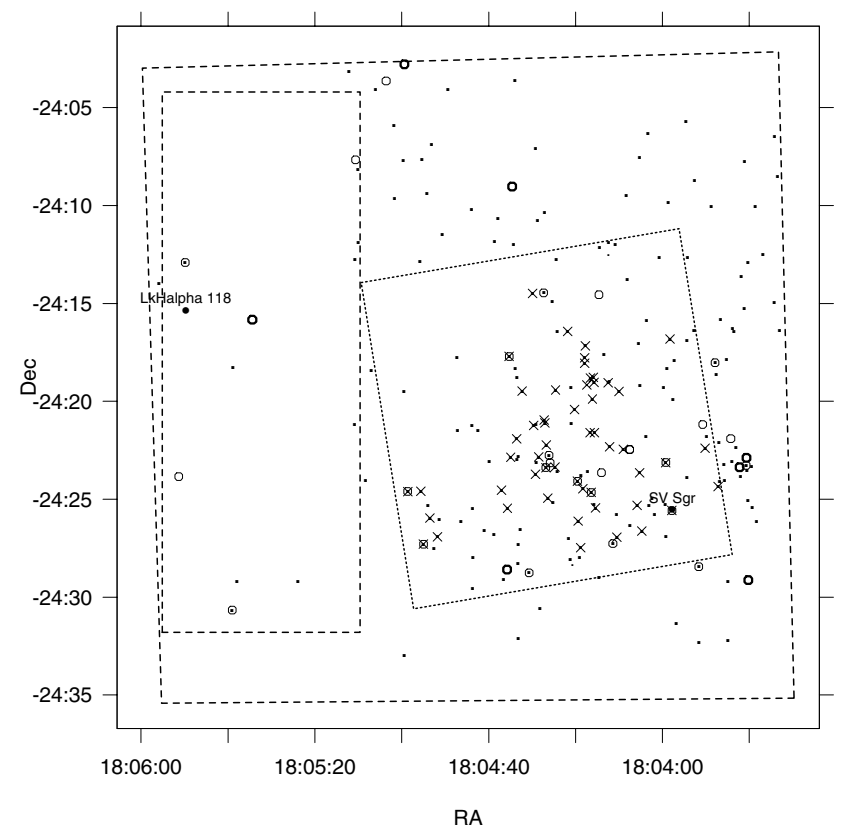

Fig. 7. Map of all stars in the region showing significant $Q_{V I J K}$ (dots) or $Q_{J H H K}$ excesses (empty circles). $\times$ 's are X-ray detected $Q_{V I J K}$-excess stars. The two labeled big dots are the only two stars in this region listed in the Herbig \& Bell (1988) catalog of PMS stars. A density gradient of $Q_{V I J K}$-excess stars can be seen across the WFI FOV, decreasing from northwest to southeast. Thick empty circles are optically undetected $Q_{J H H K}$-excess objects.

IR-excess index, not detected in X-rays, are 257 in the WFI FOV and 120 in the ACIS FOV. The number of members selected by all indicators simultaneously is only 5 for the WFI FOV (except for X-rays) and 3 for the ACIS FOV (including X-rays). The numbers in Table 1 do not always sum as expected: for example, by summing the number of members found by X-rays only (807) and that of members found in the ACIS FOV by any index $Q$ (196), one obtains 1003 , not the total value tabulated under "any $Q$ or X-rays" of 1020 members. This is due to the multiple X-ray-2MASS identifications (13 double and 2 triple), as found in Paper I.

\section{The nature of IR-excess sources}

We study here various properties of the subsamples selected above through optical-IR excess indices, in order to obtain clues to the nature of these objects. We examine space distributions, positions in color-magnitude and color-color diagrams, relationships between different optical-IR excess indices, and between these indices and accretion indicators.

\subsection{Space distributions}

In this section we examine the spatial distributions of the different classes of IR-excess stars we have found. First, we show in Fig. 7 all stars with a significant $Q_{V I J K}$ or $Q_{J H H K}$ excess. The space region considered is the whole WFI FOV, comprising the ACIS and Check fields. Only those X-ray sources showing $Q_{V I J K}$ excess are shown. It is obvious that $Q_{J H H K}$ excess sources are largely concentrated inside the ACIS FOV (18 out of 33 in the whole WFI FOV), with a smaller localized subgroup around the very young star Herschel 36, just to the right of the ACIS FOV (where two candidate protostars are also found).

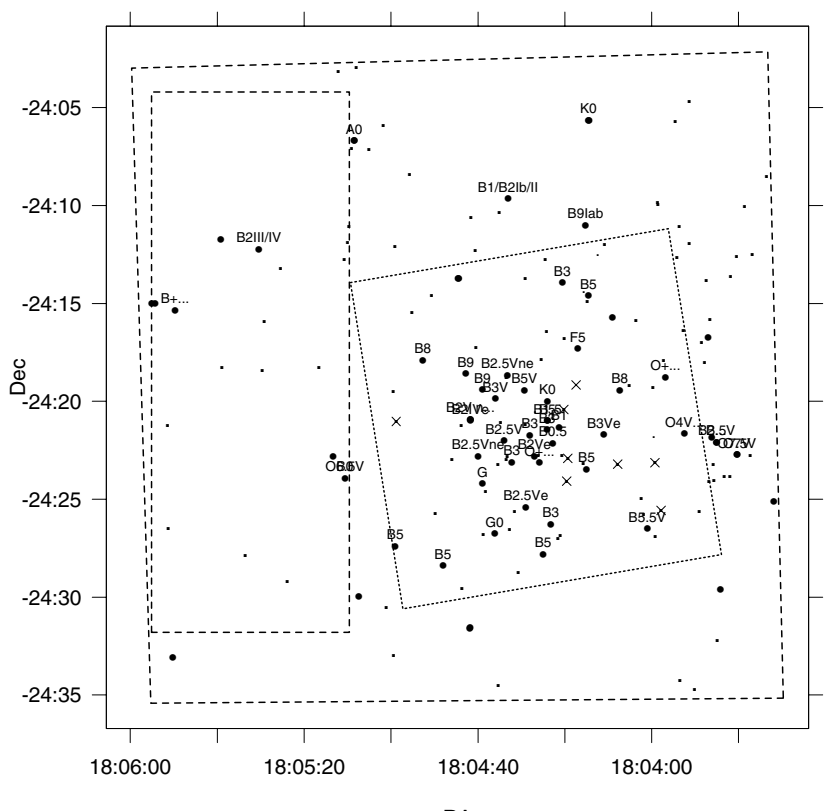

RA

Fig. 8. Map of all stars in the region showing significant $Q_{V I I J}$ excesses (small dots), with an overall similar distribution as $Q_{V I J K}$-excess stars in Fig. 7. Big dots are all stars from the Simbad database with $V<11.5$, with indicated spectral types if available. $\times$ 's are X-ray detected $Q_{V I I J}$-excess stars (much less numerous than X-ray detected $Q_{V I J K}$-excess stars in Fig. 7).

Within the ACIS FOV, $Q_{J H H K}$ excess stars are preferentially found in the lower (southern) part, precisely where younger X-ray selected cluster stars were found (Papers I and II). This is not surprising.

Less expected is instead the distribution of $Q_{V I J K}$ excess stars, which are concentrated not only around cluster center (as determined from X-ray sources, Paper I), but even more in the northwest quadrant of the ACIS FOV and in its neighborhood (even outside ACIS FOV). This region has a low average extinction, compared to other regions containing IR-excess stars (Appendix C). Only very few $Q_{V I J K}$ excess stars in this northwest region have a matching X-ray source, and in fact the X-ray source density was found in Paper I to decrease in this direction with respect to cluster center, just the opposite trend compared with the increasing density of $Q_{V I J K}$ excess stars. Therefore, this newly found IR-excess population of NGC 6530 is entirely unexpected from previous evidence. To the east and south of the ACIS FOV, instead, the density of $Q_{V I J K}$ excess stars drops drastically, in agreement with what is expected from the X-ray study in Paper I.

The fact that some IR-excess cluster members can be found even at a large distance from the cluster center or from Herschel 36 should not be considered implausible: in the figure we also report the position of the (only) two PMS stars reported in the area by Herbig \& Bell (1988), one of which $(\mathrm{LkH} \alpha 118)$ lies about 20 arcmin east of cluster center.

A similar map, but showing the distribution of $Q_{V I I J}$ excess stars, is shown in Fig. 8. The density enhancement in and around the upper right quadrant of the ACIS FOV is visible here, too, so is the gradient going from this region towards east and south, although doing so less evidently than in Fig. 7. The number of X-ray sources matching a $Q_{V I I J}$ excess star is evidently smaller than those matching $Q_{V I J K}$ excess stars, as already noted. This figure also shows the stars brighter than $V=11.5$ from SIMBAD, most of which are massive OB cluster members, 


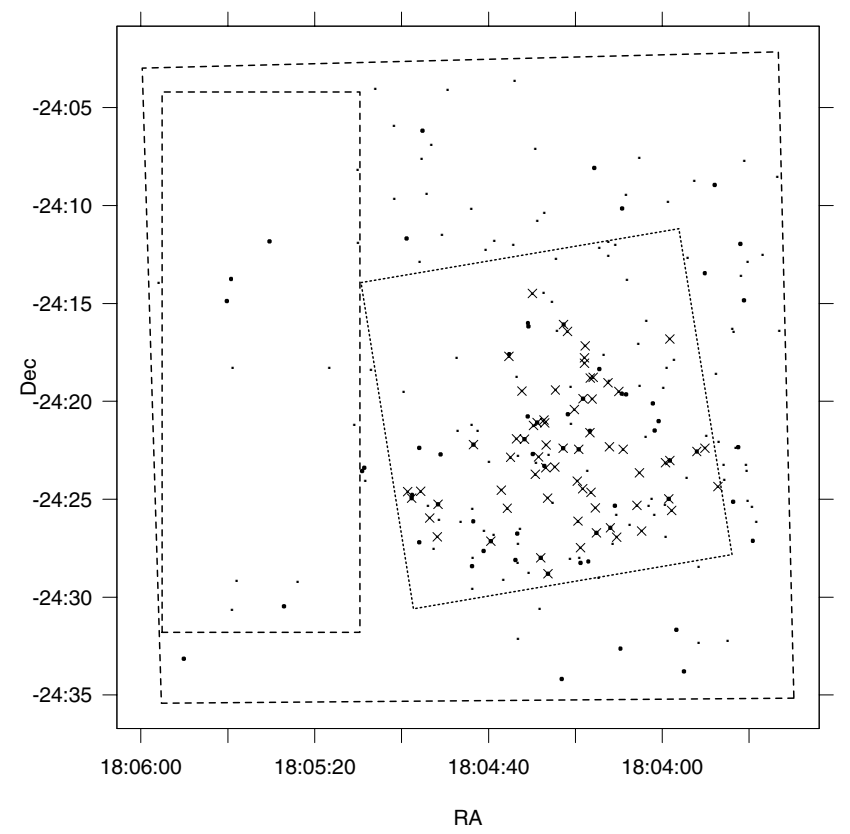

Fig. 9. Spatial map of stars with significant $Q_{B V J K}$ excess. $\times$ 's are X-ray detected stars with $Q_{B V J K}$ excess. Big dots are stars with $Q_{B V J K}$ excess, but showing no excess in any of the $Q_{V I J K}$ and $Q_{V I I J}$ indices. The $Q_{B V J K}$ excess stars are correlated more with X-ray detected stars and more concentrated towards the ACIS FOV than the $Q_{V I J K^{-}}$or $Q_{V I I J}$-excess stars (cf. Figs. 7 and 8).

as shown by the spectral types, indicated when available. These are evidently very concentrated around the center of the ACIS FOV, i.e. the X-ray determined cluster center (Paper I). On the other hand, only a few of them are found in the northwest region where the density enhancement of $Q_{V I J K}$ and $Q_{V I I J}$ excess stars is found.

Figure 9 shows the spatial distribution of $Q_{B V J K}$ excess stars. The $Q_{B V J K}$ excess stars (in particular, those showing only a $Q_{B V J K}$ excess) are more concentrated inside the ACIS FOV than the other IR-excess stars of Figs. 7 and 8. They are also more frequently coincident with X-ray detected stars (Table 1) than are the $Q_{V I J K}$ or $Q_{V I I J}$ excess stars. This suggests that the $Q_{B V J K}$ excess stars are tracing the same type of cluster stars as X-ray sources and, at the same time, that in most cases the $B$ band excess arises from enhanced chromospheres (if the X-ray activity has a coronal origin). The density of excess stars in the upper right quadrant is not higher than in the ACIS FOV, although also in this case the density to the east and south of the ACIS FOV is distinctly lower.

\subsection{Color-magnitude and color-color diagrams}

By comparing the optical and IR color-magnitude diagrams of $Q_{V I J K}$ excess stars with that of X-ray selected cluster members, we obtain clues to the nature of their photometric excess. We find that these two subsamples mostly occupy the same region in the $(V, V-I)$ diagram (not shown for brevity). Below $V=20$, some $Q_{V I J K}$ excess stars are found closer to the main sequence than to the 1-2 Myr isochrones. The $Q_{V I J K}$ excess stars are not found among highly reddened (background) stars with high $V-I$, confirming that they are not background objects, but have a reddening comparable to other cluster members. In the $(J, J-K)$ diagram, instead, $Q_{V I J K}$ excess stars are found at distinctly redder $J-K$ values than X-ray members, where they

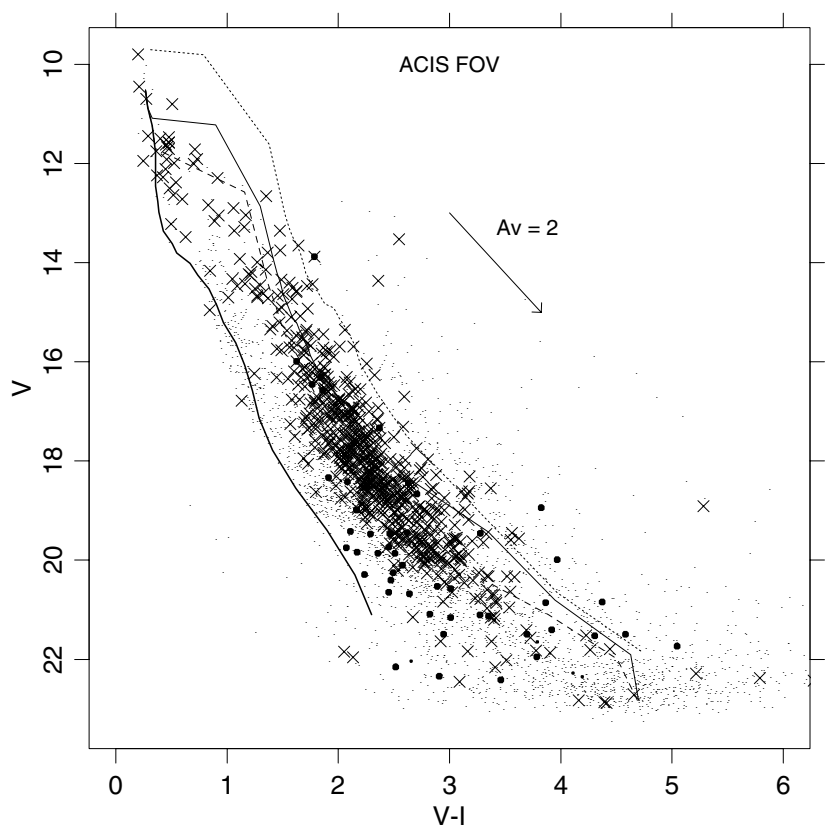

Fig. 10. $(V, V-I)$ color-magnitude diagram for stars in the ACIS FOV, showing stars with $Q_{V I I J}$ excess (big dots). X's are X-ray sources. Isochrones at $0.3,1$, and $2 \mathrm{Myr}$ are shown, as well as the ZAMS, at cluster reddening. A reddening vector is also shown.

are mixed with reddened background stars. Overall, the large overlap of $Q_{V I J K}$ excess stars with cluster members suggests that their $V-I$ colors are normal, while only their $J-K$ colors exhibit excesses. These IR excesses could not be found from a pure IR color-color diagram, such as $(J-H, H-K)$, where the bulk of these stars overlap with reddened field stars and cannot therefore be selected using the $Q_{J H H K}$ index. The spectral distribution of $Q_{V I J K}$ excess stars in the $J H K$ bands is nearly the same as a normal late-type photosphere with heavy reddening. While this coincidence may appear suspicious, we confirm the significance of this result in Appendix A.

We also examined the $(V, V-I)$ diagram of $Q_{V I I J}$ excess stars, shown in Fig. 10. In this figure we also show isochrones at ages of $0.3,1$, and $2 \mathrm{Myr}$, and the ZAMS, from Siess et al. (2000). The $Q_{V I I J}$-excess stars again show an overlap with the $\mathrm{X}$-ray detected stars, but there is also a number of them closer to the main sequence, creating a more pronounced effect than considering the $Q_{V I J K}$ excess stars. Again, very few $Q_{V I I J}$ excess stars appear to belong to the background or to be affected by strong reddening. The closeness of so many $Q_{V I I J}$ excess stars to the main sequence is puzzling: it cannot be due to a larger reddening than estimated, since correcting for more reddening would take these stars even closer to the main sequence. A naive conclusion would be that these stars are on average older than the X-ray detected members, and nevertheless have significant excesses in the near IR; alternatively, there may be excesses in the $V$ band (of the same nature as those in the $B$ band), producing a bluer $V-I$ color than the star alone. Other possibilities will be discussed on the basis of more data presented below.

Both $Q_{V I J K}$-excess and $Q_{V I I J}$-excess stars are shown in the $(J-H, H-K)$ diagram of Fig. 11, together with X-ray sources and remaining objects. The figure shows clearly that the bulk of X-ray sources has rather normal IR colors, with little reddening or IR excesses. Those X-ray sources showing an IR excess (mostly in the $Q_{V I J K}$ index) are located along the strip (or "T Tauri star locus") already noted in Paper I. Another 


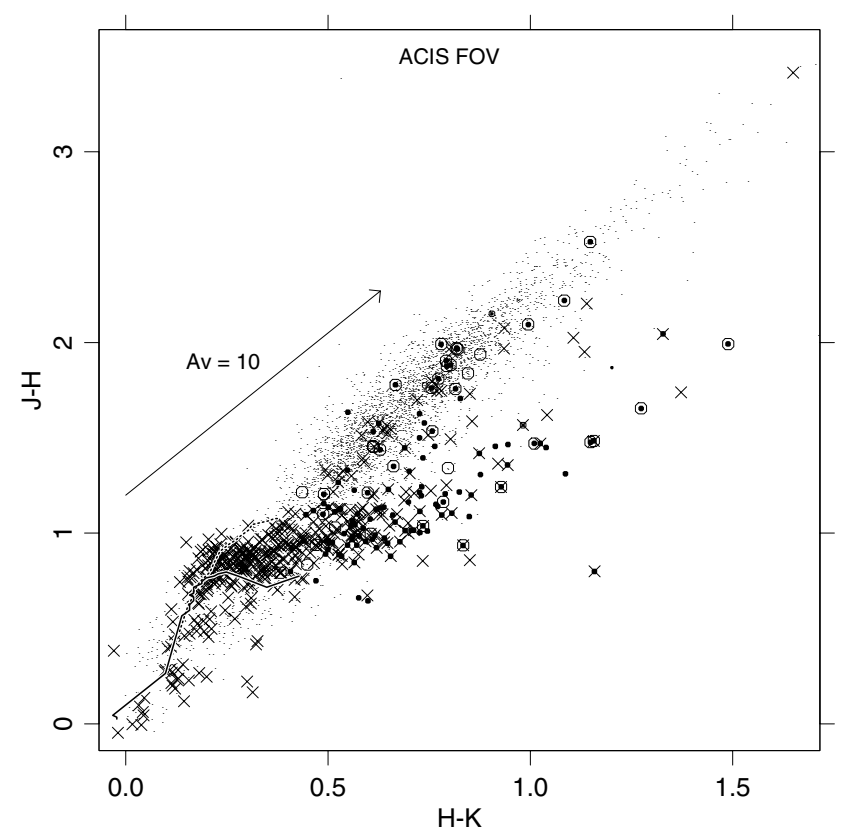

Fig. 11. $(J-H, H-K)$ color-color diagram for the ACIS FOV. The ZAMS locus and reddening vector are shown. The dotted line is the locus of giant stars. $\times$ 's are X-ray detected sources. Big filled dots are $Q_{V I J K}$-excess stars, and big empty circles are $Q_{V I I J}$-excess stars.

big group of IR excess stars (often showing both $Q_{V I J K}$ and $Q_{V I I J}$ excesses) falls instead on the "reddened main-sequence" strip. These two large concentrations of IR-excess stars suggest a "bimodal" distribution, with one group dominated by stars with X-ray emission and $Q_{V I J K}$ excess and the other by stars with $Q_{V I J K}$ and $Q_{V I I J}$ excesses. These latter stars, despite falling on the "reddened main-sequence" strip, are definitely not highly reddened normal stars: the implied reddenings would be $A_{\mathrm{V}} \sim 10$, but de-reddening them by such $A_{\mathrm{V}}$ in the $(V, V-I)$ diagram (cf. Fig. 10) would take them to implausible locations in both magnitude and color.

The color-magnitude diagram of stars with $Q_{B V J K}$ excess (not shown here) demonstrates that these stars are displaced towards bluer colors with respect to the 1-2 Myr isochrones and to the bulk of X-ray members, and thus suggests that $Q_{B V J K}$ excess stars are likely to have a blue excess, as well as an IR excess. It is very unlikely that these stars are older than other cluster stars, as the mere position in the diagram would suggest (namely, without a $B$ excess but only with a $J-K$ excess), since we do not expect an IR excess to only appear at older ages and to be lacking in younger stars. Therefore, we will not rely on the position in the HR diagram to infer ages and masses for stars considered in this paper, all characterized by excesses in their spectral energy distributions at both IR and optical wavelengths.

\subsection{Relationships between different optical-IR indices}

By comparing the two indices $Q_{V I I J}$ vs. $Q_{V I J K}$ (Fig. 12) directly, we obtain more clues to the nature of the excess-emission stars. In this reddening-free representation, deviations from the ZAMS locus are only caused by spectral peculiarities, not reddening. For the entire sample, no one-to-one relation is found between $Q_{V I I J}$ and $Q_{V I J K}$, indicating a variety of spectral distributions of excess emission. Apart from confirming the bimodal behavior already found in Fig. 11, Fig. 12 shows that those stars with both $Q_{V I J K}$ and $Q_{V I I J}$ excesses (mostly X-ray undetected)

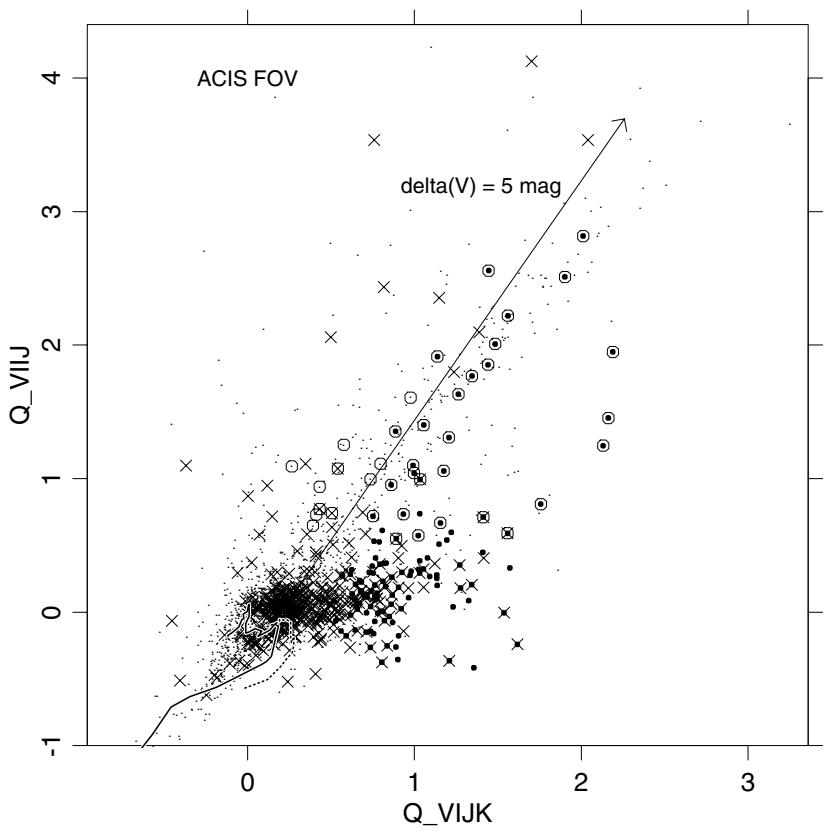

Fig. 12. Diagram of indices $Q_{V I I J}$ vs. $Q_{V I J K}$ for the ACIS FOV. The thick solid line is the ZAMS. The dotted curve is the locus of giant stars. X's are X-ray detected stars. Filled dots are $Q_{V I J K}$-excess stars, while empty circles are $Q_{V I I J}$-excess stars. The arrow is the displacement caused by a 5-mag excess only in the $V$ band. The subset of stars having both excesses shows an apparent correlation.

show a positive correlation between the $Q_{V I J K}$ and $Q_{V I I J}$ indices. Such a $Q_{V I J K^{-}} Q_{V I I J}$ correlation might either be indicative of some characteristic shape of the excess spectrum or be due to a very strong excess emission (by 2-4 mag, as shown in the figure) in the $V$ band alone.

\subsection{The puzzle of $Q_{V I I J}$-excess stars}

A $Q_{V I I J}$ excess may arise because of either a $V$-band or a $J$-band excess with respect to the $I$ band. A $V$-band excess is found for some PMS stars ("veiling") and attributed to emission from hot accreting material falling on the stellar surface. Its intensity may be comparable to the photospheric continuum, or up to $\sim 10$ times larger in the most extreme cases (e.g. Hartigan et al. 1995). To account for the values of $Q_{V I I J}$ that we observe in NGC 6530, a $V$ excess of up to $\sim 2-4 \mathrm{mag}$ in $V$ would be required, i.e. $\sim 6-40$ times the photospheric continuum, namely a very strong veiling, and the number of such heavily veiled stars in NGC 6530 would be much larger than in other regions. Moreover, if the $I-J$ color is normal, the position of $Q_{V I I J}$-excess stars in Fig. 4 indicates very large reddenings, up to $A_{\mathrm{V}} \sim 5-10$.

We have made a direct comparison with two better studied star-forming regions, Taurus-Auriga and the Orion Nebula Cluster (Fig. 13). Optical and JHK data were taken from Kenyon \& Hartmann (1995) for Tau-Aur stars and from Hillenbrand (1997) and Hillenbrand et al. (1998) for Orion stars. In both these regions, the $(V-I, J-K)$ and $(J-H, H-K)$ diagrams show many stars with $Q_{V I J K}$ and $Q_{J H H K}$ excesses. However, very few stars show a $Q_{V I I J}$ excess in the $(V-I$, $I-J$ ) diagram, unlike stars in NGC 6530 (cf. Fig. 4): there are only 2 strong $Q_{V I I J}$-excess stars $(I-J>3.5)$ in Tau-Aur, compared to 27 such stars in NGC 6530 (only in the ACIS FOV).

Although very little can be said to discriminate $V$-band from $J$-band excesses using our data, statistical considerations 

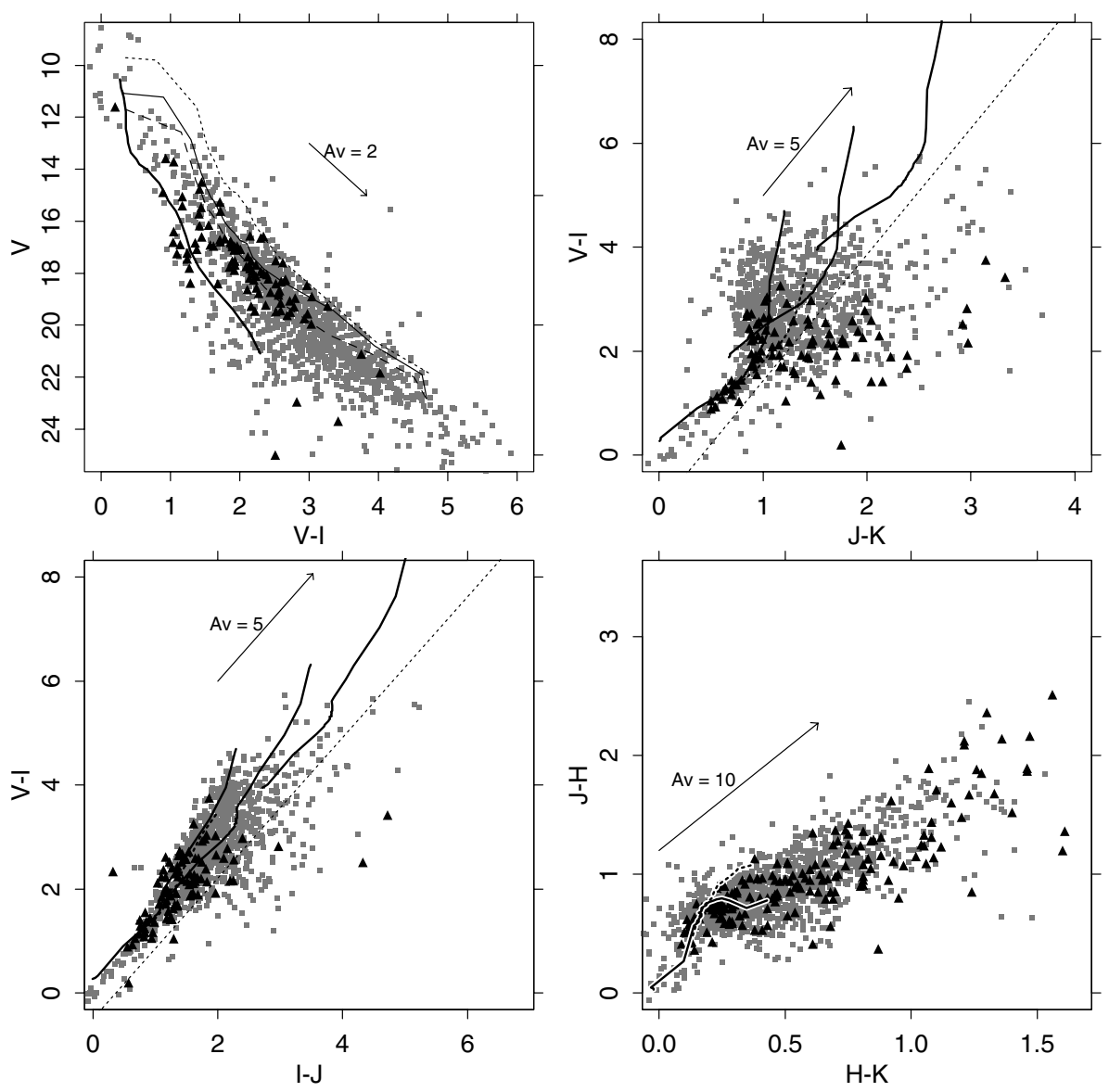

Fig. 13. Color-magnitude and color-color diagrams for PMS stars in Taurus-Auriga (black triangles) and Orion Nebula Cluster (grey squares). In the $(V, V-I)$ and $(J, J-K)$ colormagnitude diagrams, magnitudes are scaled to the distance of NGC 6530 for easier comparison. Compare with Figs. 10, 3, 4, and 11. Isochrones and other reference lines are as in these figures, respectively. In the $(V-I, I-J)$ diagram, the two black triangles at $I-J>4$ and $V-I>2$ ) are the Class I sources IRAS 04016+2610 and IRAS 04303+2240 (see text). may help. A comparison between the two color-magnitude diagrams $(I, V-I)$ and $(I, I-J)$, in particular, can show whether $Q_{V I I J}$-excess stars deviate from the locus of cluster stars more towards bluer $V-I$ or towards redder $I-J$ colors. In our data, the largest deviations are found towards redder $I-J$, but also slightly bluer than normal $V-I$ colors are found (with many stars at $I>18$ apparently near the main sequence), suggesting both types of excesses at the same time.

In order to test whether the emission in the $I$ band is photospheric, unlike that in $J H K$ bands (disk), we have examined a $(I-J, J-K)$ diagram (not shown). Under this hypothesis, the $I$ band flux (and $I-J$ ) would be uncorrelated with the $J-K$ color. However, this is not the case: most $Q_{V I I J}$-excess stars (especially strong-excess ones) show correlated $I-J$ and $J-K$ colors with a slope close to the reddening line, suggesting highly reddened $\left(A_{\mathrm{V}} \sim 10\right)$ photospheric colors from $I$ to $K$ bands. Only the $V$ magnitude would thus be discrepant with respect to all other colors, a discrepancy too large to be attributed to veiling. It also cannot be attributed to wrong identifications (Appendix A). If we de-redden the magnitudes and colors of $Q_{V I I J}$-excess stars with $V \sim 22$ (Fig.10) by $A_{\mathrm{V}} \sim 10$, we would end up with stars at $V \sim 12$ with $V-I$ colors bluer than an $\mathrm{O}$ star, which is impossible.

A possible explanation for the strong $Q_{V I I J}$-excess stars might be that the $V$ band emission is subjected to a different reddening than the longer-wavelength IJHK emission. The object producing the $V$ emission must be physically related to that (heavily reddened) emitting at $I J H K$, since the probability of a random coincidence is small (Appendix A). Such an object might be a reflection nebula near a star with a thick but non-isotropic envelope around it: the star is seen through the dusty envelope (causing a large reddening) at $I J H K$, but only through its reflected emission at $V$, if the path between the star and the nebula is much less extincted than it is towards us. Objects of this type have been found in nearer star-forming regions such as Tau-Aur, where it was possible to resolve them spatially with HST (Burrows et al. 1996; Padgett et al. 1999) or large ground-based telescopes, such as Subaru (e.g. Ishii et al. 2004). This interpretation is supported by the fact that the two strong $Q_{V I I J}$-excess stars found in Tau-Aur (Fig. 13) are indeed the Class I sources IRAS 04016+2610 (at higher $V-I$ ) and IRAS $04303+2240$, optically detected as reflection nebulosities (Padgett et al. 1999), with a lower extinction than the central object (Ishii et al. 2004) and with forbidden ([O I], [N II]) optical lines indicating extended low-density envelopes (Kenyon et al. 1998). Both these objects are optically detected at very faint $V$ magnitudes relative to their $V-I$ colors, making them to appear very close to the main sequence on a $(V, V-I)$ diagram, very similarly to our $Q_{V I I J}$-excess objects.

Also supporting this hypothesis for our $Q_{V I I J}$-excess stars, we have found that they have a distribution of apparent sizes (as indicated by the "sharpness" parameter from the DAOPHOT software, Stetson 1987) that is wider than that of all optical stars with a 2 MASS identification (Fig. 14), with a significant tail at high values indicating marginally extended objects, unlike stars in the whole sample.

Therefore, we may interpret the $Q_{V I I J}$-excess stars with two alternative scenarios: Case (a), or "filled-hole disk", favored for weak- excess stars: low-extinction cluster members, surrounded by thick disks, reaching inward close to the star, with unusually small inner holes, and producing moderate $V$ excesses due to the accretion continuum (veiling); the spectral energy distribution 

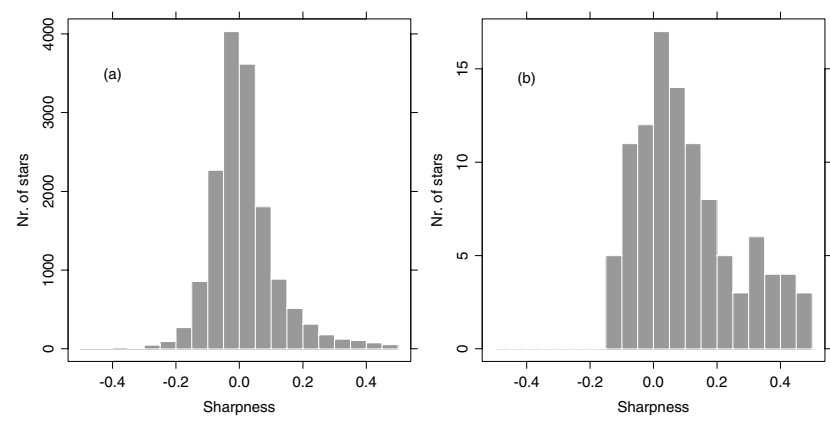

Fig. 14. Distribution of "sharpness" for all WFI optical stars with a 2MASS identification a) and for $Q_{V I I J}$-excess stars b).

of such disks happens to mimic well a heavily reddened normal photosphere from the $I$ band up to the $K$ band. In Case (b), or "reflection nebula", favored for strong-excess stars, highly reddened cluster members are partially surrounded by a thick envelope, but without strong emission from the disk or envelope, so that normal, reddened stellar colors are observed in the range between the $I$ and $K$ bands; their discrepant $V$ emission arises from a nebula (size on the order of $1000 \mathrm{AU}$ ), reflecting the light from the central star with little extinction.

\subsection{IR excesses and accretion}

We studied whether the circumstellar disks found by their optical-IR excesses are actively accreting matter onto the star. Classical indicators of accretion are usually the $\mathrm{H} \alpha$ equivalent width or UV excesses. For a subsample of our stars limited to $V<17$, and covering our ACIS FOV only partially, $U$ band data and the $R-\mathrm{H} \alpha$ index (proxy for the $\mathrm{H} \alpha$ equivalent width) are given by SCB. The existence of accretion is perhaps a necessary ingredient to support the validity of our picture of filledhole disks. Such disks would have their inner regions rapidly destroyed by dust photoevaporation, because of the strong stellar irradiation (the dust destruction temperature is around $1400 \mathrm{~K}$ ); therefore, they could not exist unless their inner parts are continuously replenished by material which spirals in and accretes onto the star.

Figure 15 is a diagram of the Johnson $Q$ (i.e., $(U-B)-$ $0.72(B-V))$ index vs. our $Q_{V I J K}$ index with stars with $Q_{V I J K}$ $\left(Q_{V I I J}\right)$ excess. In such a reddening-free diagram, deviations from normal photospheric colors are uniquely due to excess emission. A UV excess lowers the Johnson $Q$ index with respect to its photospheric value. From the observed $V-I$ color we may predict the expected position of each star in this diagram. For $Q_{V I J K}$ and $Q_{V I I J}$ excess stars, arrows connect such expected positions to the actually observed values, which are in many cases strongly discrepant with the expected ones. The horizontal component of an arrow represents therefore the IR excess of a star, while the vertical (downward) component is its UV excess. Such a representation shows that, although many $Q_{V I J K}$-excess stars share with normal stars a common range of Johnson $Q$ values, their individual $Q$ values corresponding to their $V-I$ colors do not agree with the observed values, as indicated by the systematically downward slopes of all plotted arrows. Therefore, all stars showing an IR excess, defined by our $Q_{V I J K}$ or $Q_{V I I J}$ indices, show some UV excess (from less than 0.5 mag up to $1.5 \mathrm{mag}$ ), most likely due to accretion. A qualitatively similar result is obtained by considering the $R-\mathrm{H} \alpha$ index (itself reddening-free), which is found to be

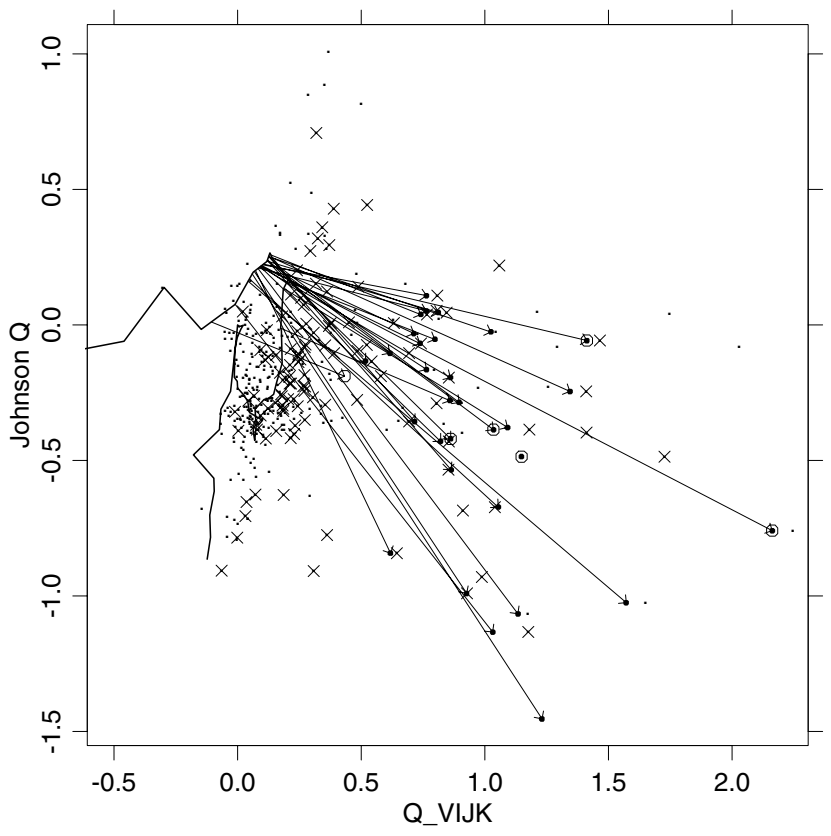

Fig. 15. Diagram of the Johnson $Q$ index vs. our $Q_{V I J K}$ index. Stars with $Q_{V I J K}$ excess are indicated by big filled dots, while stars with $Q_{V I I J}$ excess are indicated by empty circles. $\times$ 's are X-ray sources, and small dots all remaining stars with $U$ band data. Arrows indicate the observed displacement of data points, with respect to their expected position from the $V-I$ color. The thick solid line indicates ZAMS colors.

positively correlated with our $Q_{V I J K}$ index. One object (SCB \#625) shows extreme values of both these indices.

\section{Discussion}

Using the results found in the previous sections, we discuss here some of their implications on the observed frequency of circumstellar disks in NGC 6530, on the relationship found between disks and X-ray emission, and last on the disk evolution in different regions of the cluster, under different ambient conditions.

\subsection{Disk frequency and types}

Analysis of the combined optical and IR colors has enabled us to select an additional population of stars with excesses in their optical-IR spectral energy distribution (257 X-ray undetected stars, of which 120 in the ACIS FOV). They are very good candidates to be NGC 6530 cluster members, which add to the large X-ray selected population of Papers I and II. From the numbers of probable cluster members found above using different methods, we can estimate the frequency of circumstellar disks in NGC 6530. All our X-ray, optical, and IR data are flux-limited, implying that the derived fraction is computed for stars more massive than $\sim 0.5 M_{\odot}$ (Paper II). From the number of all IR-excess stars (therefore, with disks) in ACIS FOV and the number of all members in the same field, we derive a disk frequency of $196 / 1020=19.2 \%$; unfortunately, only in the ACIS FOV do we have membership indicators other than IR to obtain the total number of members. Both the numerator and the denominator of this fraction should be lowered slightly, to account for a fraction $(\sim 10 \%)$ of "chance coincidences" in the IR-excess members, as discussed above, and for a fraction of non-member X-ray sources $(\sim 5-10 \%$, Paper I), so that the final disk frequency should be still on the order of $20 \%$. This value 
is much lower than found in the Orion Trapezium Cluster (80-85\%) by Lada et al. (2000), who however use the $L$ band data that are more sensitive to disk emission.

The most significant result of this study, however, is not just a "numerical" enrichment of the known cluster population, but the fact that the newly selected cluster stars show many properties that differ from those of the already known members. In discussing the properties of subsamples showing different optical-IR excesses, it is convenient to treat stars with weak and strong $Q_{V I I J}$ excesses differently (defined in Fig. 4), interpreted respectively as "filled-hole disk" and "reflection nebula" objects, from the discussion in Sect. 5.

\subsection{X-rays and disks}

We noted the intriguing "bimodal" distribution of cluster stars of Fig. 12. All of the stars in this figure fall in the X-ray observed ACIS FOV, but those with a $Q_{V I J K}$ excess are detected in X-rays much more frequently than those with a $Q_{V I I J}$ excess (X-ray detection fractions of $42.7 \%$ and $15.1 \%$, respectively). We recall that the former group has a $K$ band (and some $H$ band) excess predominantly, while the second group has significant excess in the $J$ band (filled-hole disks) $o r$ in the $V$ band (reflection nebulae).

By considering strong and weak $Q_{V I I J}$-excess objects separately, however, we find significantly different X-ray detection fractions of $3.7 \%(1 / 27)$ and $27 \%(7 / 26)$, respectively. The latter fraction is not significantly different from that of $Q_{V I J K}$-excess stars $(42.7 \%)$, so the subsample with filled-hole disks does not appear to show a reduced X-ray emission with respect to other optical-IR excess stars (with larger inner disk holes). The reduced average X-ray detection rate of $Q_{V I I J}$-excess objects must instead been attributed to the very low X-ray detection rate of the "reflection nebula" candidates (only one X-ray detection, source \#311, among the 27 objects in the ACIS FOV). This can be explained naturally, since these objects have a very high extinction along the line of sight. They are candidate Class I objects, which are sometimes detected in X-rays in less distant starforming regions (e.g. $\rho \mathrm{Oph}$ ).

\subsection{Spatial differences}

A different issue is related to the spatial distribution of the $Q_{V I J K}$ and $Q_{V I I J}$ excess stars, found to be different from that of X-ray detected stars and of bright $(\mathrm{OB})$ cluster stars, in the sense that the new IR-excess stars are concentrated significantly towards the northwest of the cluster center. In Paper I, we found a difference in the ages of X-ray detected cluster stars, with older stars in the northwest part of the ACIS FOV and younger ones in the south. Although the subsample of X-ray detected IR-excess stars is small, especially in the northwest part of the ACIS FOV (Figs. 7 and 8), the spatial location of optical-IR excess stars is similar to that of the older cluster stars. Since our optical-IR excess stars may have excesses in the optical bands, not just in the IR bands, it is not safe to derive ages from their position in the HR diagram. Even though their apparent position in the diagram is sometimes close to the main sequence (e.g. Fig. 10), objects with circumstellar accretion disks are expected to be very young (and reflection nebulae even more so), and their co-spatiality with older stars may appear strange, especially in a region such as NGC 6530, where well-defined age gradients have been claimed (Lada et al. 1976, Papers I, II). In other regions such as Tau-Aur, however, we are accustomed to finding protostellar objects not far from more evolved weak-line T Tauri stars, making it likely that star formation occurs non-coevally even within the same piece of cloud. This is probably the case even for NGC 6530, at least in its northern parts.

The fact that objects with conspicuous disks are numerous in the north of NGC 6530, where star formation appears to have started long ago with respect to e.g. the southern regions where only very young stars are found, may also reflect that disks around these stars are longer-lived than their analogues around stars near cluster center/southern parts. As is obvious from Fig. 8, only very few massive B stars are found in the northwest region, and thus stars in this region receive a much smaller ionizing flux than stars near cluster center, and their surrounding material is also subject to a much weaker dynamical pressure from high-mass stars' winds. There is ample evidence that radiation and wind pressures from massive stars (e.g. from $\theta^{1}$ Ori in the Orion Nebula Cluster) act efficiently to (photo)evaporate disks (the so-called proplyds observed by HST; O'Dell et al. 1993; O'Dell 2001); the evolution of undisturbed disks would probably proceed on longer time scales. Therefore, there would be no unique disk evolution time scale, but this would instead be very sensitive to the ambient conditions, especially the presence of neighboring massive stars. Even some main-sequence stars still have tenuous disks. We note that the disk frequency in the giant starburst star-forming region NGC 3603 (1 Myr old) was recently found by Stolte et al. (2004) to vary from $20 \%$ to $40 \%$ in going from cluster center to its external parts, and this was interpreted as due to strong photoevaporation near the cluster center, in qualitative agreement with our picture just discussed. At the other extreme, in the low-density region of $\eta$ Cha, Lyo et al. (2003) find that disks may even last for about 10 Myrs.

The picture that our data suggest for NGC 6530, therefore, is that stars in the northwest region formed over a long time span, since early epochs during the cluster evolution, but very few massive stars were born in this region, so that the evolution of circumstellar disks around these stars was slow; then, stars formed near the cluster center (and in southern regions) with perhaps greater efficiency, including many massive stars still present today (Fig. 8). These caused a faster evolution of disks, and clearing out of their inner regions, thus permitting more active X-ray coronae to develop.

\section{Summary and conclusions}

In this study we have made an in-depth comparison of available X-ray, optical, and infrared data on stars in the region of the very young cluster NGC 6530, to complement our X-ray study of Paper I and our optical study of Paper II. Different nonstandard combinations of optical and IR colors in the form of reddening-free indices were used to select more cluster members, showing IR excesses, thereby finding 257 new probable members (not previously detected in X-rays) over an approximately $33^{\prime} \times 33^{\prime}$ region comprising the ACIS field studied in Paper I, where more than 800 candidate members had already been found.

The new cluster members found by different methods show different average characteristics. While X-ray detected stars, massive stars, and stars with blue excess tend to be co-spatial, stars with optical-IR excesses show a higher concentration in a different region, northwest of the previously determined cluster center. Furthermore, optical-IR excess stars show a bimodal distribution of spectral energy distributions, depending largely on whether they show an excess in the $Q_{V I I J}$ index (namely, in the $J$ or $V$ band) or not. Stars with a $Q_{V I I J}$ excess are much 
less frequently detected in X-rays than stars with only longerwavelength ( $K$ band) excesses. This absence of X-ray emission is particularly evident for a subsample of objects, whose optical-IR excess is best interpreted as a $V$ band excess flux (with respect to a very reddened star), coming from a reflection nebulosity close to the star. Such reflection nebulae around very young PMS stars are well known in nearer star-forming regions.

A definitive confirmation that strong $Q_{V I I J}$-excess stars are indeed reflection nebulae, as we suggest, might be obtained by high-resolution imaging. Reflection nebulae around PMS stars in Taurus were found by Padgett et al. (1999) with major axes in the range between 500-1000 AU, which at the distance of NGC 6530 correspond to $0.4-0.8$ arcsec. In case of a positive result from detailed imaging studies, our $Q_{V I I J}$ index would provide a rather simple and powerful indicator of the presence of such objects, even in distant star-forming regions.

Many optical-IR excess stars are co-spatial with a cluster sub-population of stars older than (X-ray detected) central cluster stars. We argue that this is probably an ambient-related effect, where lower ionizing fluxes and winds from massive stars either do not force a rapid disk evolution, and permit longer disk lifetimes, and/or do not prevent star formation to occur over a long time span. In addition to a time sequence of star-forming events in NGC 6530, as found in Papers I and II, the new data therefore also suggest qualitatively different star-formation episodes, a former one with low efficiency on long time scales and a more recent one, more rapid and efficient, yielding the bulk of stars (including massive ones). This would have affected the subsequent evolution of stars differently in different subregions of NGC 6530.

In order to obtain additional membership information for NGC 6530 stars, we have also obtained optical spectra of more than 300 stars (both X-ray detected and not) in the ACIS FOV, from which lithium abundances, radial, and rotational velocities are being measured (Prisinzano et al. 2006). We plan to extend this optical follow-up to IR-excess candidate members, thus obtaining a check of their actual nature, to give a more solid basis to the picture suggested here.

In any case, our study makes the census of NGC 6530 members to increase to more than 1100 stars in our WFI FOV. To these, one should add the several hundred additional X-ray sources found by Castro et al. (2004) in a new Chandra ACIS-I observation (three times longer than that analyzed by us in Paper I), centered on Herschel 36, and overlapping our WFI FOV only partially. The total number of NGC 6530 members will eventually approach 2000, namely more than the Orion Nebula Cluster, as predicted in Paper I on the basis of the massive star content. This fact, coupled with the highly differentiated features found in NGC 6530 (age gradients and maybe different regions forming low- and high-mass stars), makes this cluster a very promising target for a deeper study of star-formation processes and of the evolution of PMS stars.

Acknowledgements. We thank an anonymous referee for useful suggestions to improve the presentation of this paper. The authors acknowledge support from an Italian MIUR PRIN grant. This study has made use of the 2MASS data and of the SIMBAD database, operated at the CDS, Strasbourg.

\section{References}

Baraffe, I., Chabrier, G., Allard, F., \& Hauschildt, P. H. 2002, A\&A, 382, 563 Bertout, C., Basri, G., \& Bouvier, J. 1988, ApJ, 330, 350

Bessell, M. S., \& Brett, J. M. 1988, PASP, 100, 1134

Burrows, C. J., Stapelfeldt, K. R., Watson, A. M., et al. 1996, ApJ, 473, 437

Calvet, N., \& Gullbring, E. 1998, ApJ, 509, 802

Castro, P. J., Gagne, M., Tothill, N. F., Kenworthy, M. A., \& McCaughrean, M. J. 2004, Am. Astron. Soc. Meet., 204, 6213

Cutri, R. M., et al. 2003, Explanatory Supplement to the 2MASS All Sky Data Release, http://www.ipac.caltech.edu/2mass/releases/ allsky/doc/explsup.html

Damiani, F., Flaccomio, E., Micela, G., et al. 2004, ApJ, 608, 781 (Paper I)

Hartigan, P., Edwards, S., \& Ghandour, L. 1995, ApJ, 452, 736

Herbig, G. H., \& Bell, K. R. 1988, Lick Observatory Bulletin, Santa Cruz: Lick Observatory

Hillenbrand, L. A. 1997, AJ, 113, 1733

Hillenbrand, L. A., Strom, S. E., Vrba, F. J., \& Keene, J. 1992, ApJ, 397, 613

Hillenbrand, L. A., Strom, S. E., Calvet, N., et al. 1998, AJ, 116, 1816

Ishii, M., Tamura, M., \& Itoh, Y. 2004, ApJ, 612, 956

Kenyon, S. J., \& Hartmann, L. 1995, ApJS, 101, 117

Kenyon, S. J., Yi, I., \& Hartmann, L. 1996, ApJ, 462, 439

Kenyon, S. J., Brown, D. I., Tout, C. A., \& Berlind, P. 1998, AJ, 115, 2491

Lada, C. J., \& Adams, F. C. 1992, ApJ, 393, 278

Lada, C. J., Gottlieb, C. A., Gottlieb, E. W., \& Gull, T. R. 1976, ApJ, 203, 159

Lada, C. J., Muench, A. A., Haisch, K. E., et al. 2000, AJ, 120, 3162

Lyo, A.-R., Lawson, W. A., Mamajek, E. E., et al. 2003, MNRAS, 338, 616 Mathis, J. S. 1990, ARA\&A, 28, 37

Meyer, M. R., Calvet, N., \& Hillenbrand, L. A. 1997, AJ, 114, 288

O'dell, C. R. 2001, ARA\&A, 39, 99

O'dell, C. R., Wen, Z., \& Hu, X. 1993, ApJ, 410, 696

Padgett, D. L., Brandner, W., Stapelfeldt, K. R., et al. 1999, AJ, 117, 1490

Prisinzano, L., Damiani, F., Micela, G., \& Sciortino, S. 2005, A\&A, 430, 941 (Paper II)

Prisinzano, L., Damiani, F., Micela, G., \& Pillitteri, I. 2006, A\&A, in press

Rauw, G., Nazé, Y., Gosset, E., et al. 2002, A\&A, 395, 499

Rieke, G. H., \& Lebofsky, M. J. 1985, ApJ, 288, 618

Siess, L., Dufour, E., \& Forestini, M. 2000, A\&A, 358, 593

Steson, P. B. 1987, PASP, 99, 191

Stolte, A., Brandner, W., Brandl, B., Zinnecker, H., \& Grebel, E. K. 2004, AJ, 128,765

Sung, H., Chun, M., \& Bessell, M. S. 2000, AJ, 120, 333 (SCB)

The, P. 1960, ApJ, 132, 40

van den Ancker, M. E., The, P. S., Feinstein, A., et al. 1997, A\&AS, 123, 63

Walker, M. F. 1957, ApJ, 125, 636 
F. Damiani et al.: Chandra and 2MASS study of NGC 6530, Online Material p 1

\section{Online Material}




\section{Appendix A: Robustness of results}

A relevant source of uncertainty when matching two large catalogs, as in our case, are misidentifications between optical $(B V I)$ and IR $(J H K)$ objects due to chance coincidences. There are 53581 stars in the WFI catalog of Paper II and 34791 2MASS sources in the same area. With a maximum identification radius of $0.8 \operatorname{arcsec}^{2}$ (used after correcting for small systematic offsets between the two catalogs), we may expect 969 chance coincidences over the entire WFI FOV (out of the 15282 identifications found), if the two populations were uncorrelated (as discussed in Paper I). In the ACIS FOV, the numbers of WFI and 2MASS objects are 11996 and 9237, respectively, and the predicted (maximum) number of chance coincidences is 217 (out of 4083 identifications).

Since the large number of optical-IR identifications found implies a strong correlation between the optical stars and nearIR sources, these estimates of the number of chance coincidences must be considered as very conservative upper limits. In Appendix B we describe a refined method of computing a more realistic number of chance coincidences between two lists of positions, taking the number of coincidences actually found into account. Using this method, the expected number of chance coincidences reduces to 395 in the WFI FOV and 82 in the ACIS FOV.

We then examined to what extent chance (i.e. random) optical-IR matches can have affected our findings about objects with excess emission in the $Q_{V I J K}$ and $Q_{V I I J}$ indices. We have tried to preserve the large-scale spatial properties of our optical and IR samples (objects in different sub-regions have different average IR colors, see Appendix C), on scales larger than $\sim 1$ arcmin. We therefore shifted our WFI positions by 15 arcsec (a much larger distance than the identification radius of 0.8 arcsec, but smaller than typical scales of density variations) and "identified" these stars with the real 2MASS objects. This ensures that no real identifications are included, so we obtained 950 spurious identifications over the whole WFI FOV, very close to the expected number derived above (969), not taking the actual correlation between catalogs into account. Since the expected number of truly uncorrelated chance coincidences is 395 , as derived above, we randomly selected a subsample of this size out of the 950 spurious identifications found initially. We call these test objects "chance identifications" (CI).

We computed the various excess indices for these CI objects, and found, in the ACIS FOV, a significant $Q_{V I J K}$ excess in 6 cases (see for example Fig. A.1a) and a significant $Q_{V I I J}$ excess in 11 cases. Five objects in the ACIS FOV had both excesses. The corresponding numbers found in the actual data were 124,53 , and a common subset of 30 , so that CI may explain only a small fraction $(<10 \%$ overall) of the IR-excess stars found in the ACIS FOV. This number rises to $\sim 17 \%$ across the entire WFI FOV. Figures A.1 $\mathrm{a}$ and $\mathrm{b}$ also show tens of CI falling in the same regions as IR-excess stars, but they were not selected as such, because of larger photometric errors than we allowed. This demonstrates the need for a conservative definition of $Q_{V I J K}$ and $Q_{V I I J}$-excess stars, such as the one we adopted, in order to minimize the contamination by CI.

We placed the CI with an apparent IR excess in various diagrams among those discussed above. Figure A.1b shows the location of these CI objects (and in particular those with $Q_{V I J K}$ or $Q_{V I I J}$ excesses) in the ( $\left.V, V-I\right)$ diagram, to be compared with

\footnotetext{
2 We recall that the 0.8 arcsec radius was used to cross-identify the whole optical and IR catalogs, while we conservatively used a smaller radius of 0.4 arcsec for selecting the subset of optical-IR excess stars.
}

those of the real $Q_{V I I J}$ excess stars (Fig. 10). The CI produce very few objects at locations around the 1-2 Myr isochrones, which instead abound in the real data. They instead make some contribution at locations nearer to the main sequence at $V>19$, where also a few IR-excess stars are found in the real data of Fig. 10. Therefore, a few IR-excess stars found in this part of the diagram are possibly spurious.

We also examined the distribution of offsets between WFI and 2MASS positions, for stars in ACIS FOV showing (in the real data) a $Q_{V I J K}$ excess (allowing identifications up to 0.8 arcsec, instead of the finally adopted 0.4 arcsec limit), as shown in Fig. A.1c. This figure shows a very similar distribution to that of all other 2MASS-WFI coincidences in the ACIS FOV, and that is very different from the expected distribution of chance coincidences between unrelated catalogs (a distribution linearly increasing with offset $r$ up to the identification radius of 0.8 arcsec, since the area element is $\mathrm{d} A=2 \pi r \mathrm{~d} r$ ). The same figure shows the corresponding distribution of offsets for CI objects with $Q_{V I J K}$ excess, which are much less numerous and more frequent beyond 0.4 arcsec. Figure A.1d shows the corresponding distributions for $Q_{V I I J}$-excess stars (both real and CI). In the real data there is now no well-defined peak at small offsets: this might be a real effect related to some nebulosity around these objects (see Fig. 14 and the corresponding discussion) or simply an indication of a higher than expected contamination at large offsets. Conservatively, we retained only identifications with offsets less than 0.4 arcsec (vertical line in Figs. A.1c and d), where the expected contamination is minimal. After this final test, we can safely conclude that chance identifications between WFI and 2MASS catalogs are a definitely minor effect that cannot noticeably alter our conclusions.

\section{Appendix B: Evaluation of the expected number of spurious cross-identifications}

The number of wrong identifications expected when matching two catalogs of sky positions is usually computed under the (implicit) assumption that no true correlation exists between the two catalogs. As mentioned in Paper I, this may lead to an overestimate, sometimes by a large factor, of the actual number of random chance coincidences between the two catalogs, when a substantial correlation is indeed expected between the two sets of objects (e.g., measurements made in two adjacent wavelength bands). Therefore, here we study how a more realistic expected value for the number of chance coincidences can be computed, with no extra assumptions on the nature of the data. For simplicity, we assume that positional accuracy of the cataloged objects is good enough to yield no multiple identifications (this holds for the vast majority of objects studied in this paper).

Let us assume that the two catalogs contain $N_{1}$ and $N_{2}$ objects, respectively, distributed uniformly over the same sky area $A_{\text {tot }}$. For cross-identification, a fixed or position-dependent radius may be used, leading to a mean "area for identification" (averaged over catalog objects) $\left\langle A_{\text {id }}\right\rangle$. Now, each list contains a subset of objects, with a corresponding one in the other list, and a subset without corresponding objects:

$N_{1}=N_{1}^{\text {corr }}+N_{1}^{\text {uncorr }}$

$N_{2}=N_{2}^{\text {corr }}+N_{2}^{\text {uncorr }}$

and $N_{1}^{\text {corr }}=N_{2}^{\text {corr }}$ by definition. Therefore, the total number of identifications found between the two lists is:

$N_{\text {id }}=N_{1}^{\text {corr }}+N_{\text {spurious }}$ 

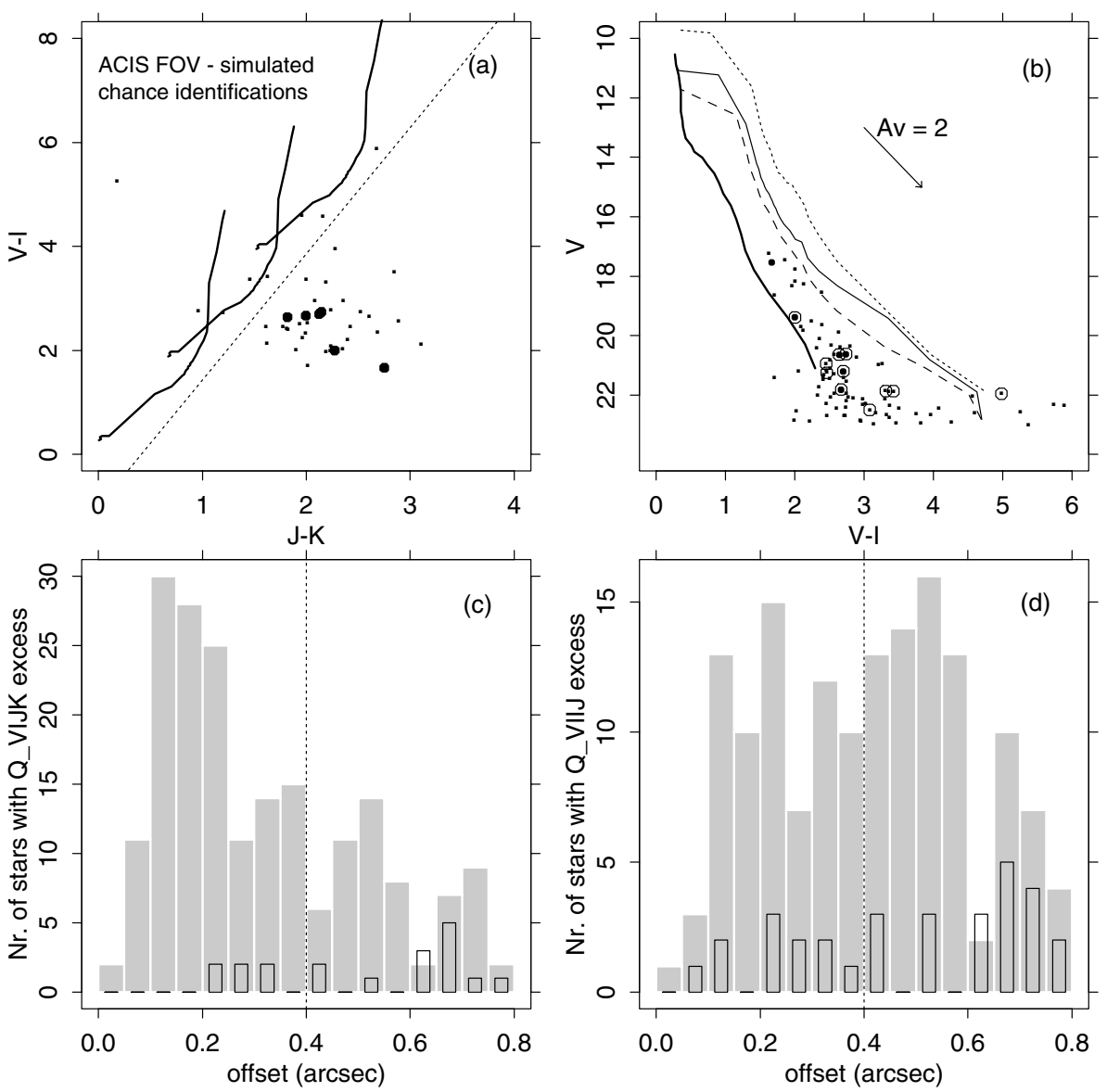

Fig. A.1. Features of simulated 2MASSWFI chance identifications (CI) in the ACIS FOV: a): $(V-I, J-K)$ diagram; big dots are $Q_{V I J K}$-excess stars in the simulated data, defined as in the real data. Isochrones as in Fig. 3 (to be compared with this figure). b): $(V, V-I)$ diagram; empty circles are $Q_{V I I J^{-}}$ excess stars in simulated data, other symbols as in panel a). c): distribution of spatial offsets between 2MASS and WFI positions, for real $Q_{V I J K}$-excess stars in the ACIS FOV (grey histogram), including stars with an offset up to 0.8 arcsec. Narrower bars indicate offsets for simulated CI with $Q_{V I J K}$ excess, again up to 0.8 arcsec. d): same as panel c) for $Q_{V I I J}$-excess stars (both real and simulated). where

$N_{\text {spurious }}=N_{1}^{\text {uncorr }} N_{2}^{\text {uncorr }} \frac{\left\langle A_{\text {id }}\right\rangle}{A_{\text {tot }}}$.

This one is the usual formula for computing the expected number of spurious identifications, taking $N_{i}^{\text {uncorr }}=N_{i}(i=1,2)$. If $N_{i}^{\text {uncorr }}$ is significantly less than $N_{i}$, the classical formula overestimates the true $N_{\text {spurious. }}$. By substitution, we have:

$N_{\text {id }}=N_{1}^{\text {corr }}+\left(N_{1}-N_{1}^{\text {corr }}\right)\left(N_{2}-N_{1}^{\text {corr }}\right) \frac{\left\langle A_{\text {id }}\right\rangle}{A_{\text {tot }}}$.

This is a second-degree equation in $N_{1}^{\text {corr }}$, easily solved as

$$
\begin{aligned}
N_{1}^{\mathrm{corr}}= & 0.5\left(\left(N_{1}+N_{2}-\frac{A_{\mathrm{tot}}}{\left\langle A_{\mathrm{id}}\right\rangle}\right)\right. \\
& \left. \pm \sqrt{\left(N_{1}+N_{2}-\frac{A_{\mathrm{tot}}}{\left\langle A_{\mathrm{id}}\right\rangle}\right)^{2}-4\left(N_{1} N_{2}-\frac{A_{\mathrm{tot}}}{\left\langle A_{\mathrm{id}}\right\rangle} N_{\mathrm{id}}\right)}\right)
\end{aligned}
$$

and eventually $N_{\text {spurious }}=N_{\text {id }}-N_{1}^{\text {corr }}$.

We have tested the reliability of this approach, trying to push it further to predict the number of double identifications between the two catalogs, and comparing the predicted number with what is actually found when matching e.g. the 2MASS and WFI source lists in NGC 6530. We take list \#1 as a reference, and define as $N_{\mathrm{id} 2}$ the number of objects in this list that are identified with $t w o$ objects from list \#2. Given an identification area $\left\langle A_{\text {id }}\right\rangle$ around each object in list \#1, the number of uncorrelated objects in list \#2 falling in this area has an expected value of $\mu=N_{2}^{\text {uncorr }}\left\langle A_{\text {id }}\right\rangle / A_{\text {tot }}$, with a Poisson distribution. The probability of finding $n$ uncorrelated objects in that area is therefore $P_{n}(\mu)$. The double idenfications will include two contributions: "true" identifications plus another "spurious" identification, and totally spurious identifications of one object in list \#1 simultaneously with two in list \#2. The total number of double identifications is thus predicted to be:

$N_{\mathrm{id} 2}=N_{1}^{\mathrm{corr}} P_{1}(\mu)+N_{1}^{\mathrm{uncorr}} P_{2}(\mu)$.

In the 2MASS-WFI cross-identification, we used an identification radius of $0.8^{\prime \prime}$ over the WFI FOV of $33^{\prime} \times 33^{\prime}$, and by the method outlined above the number of uncorrelated WFI stars is $N_{2}^{\text {uncorr }}=38694$, so that $\mu=0.0198, P_{1}(\mu)=1.94 \times 10^{-2}$, and $P_{2}(\mu)=1.93 \times 10^{-4}$. Since the numbers of correlated and uncorrelated objects in list \#1 (again derived as above) are of the same order of magnitude, $N_{1}^{\text {corr }}=14887$ and $N_{1}^{\text {uncorr }}=19904$, the first term in Eq. (B.2) is largely dominant over the second term, and we predict $N_{\mathrm{id} 2}=293$ double 2MASS-WFI identification over the whole WFI FOV, mostly due to the large number of correlated objects. This number compares very well with the actual number of double identifications found (321). If we had ignored the correlated objects, we should have applied Eq. (B.2) with $N_{1}^{\text {corr }}=0, N_{1}^{\text {uncorr }}=N_{1}=34791$, and $N_{2}^{\text {uncorr }}=N_{2}=53581$, yielding $N_{\mathrm{id} 2}=13$, which is much smaller than the number actually found. When estimating the numbers of spurious and double identifications obtained by matching two catalogs, it is therefore essential to take the correlation between the catalogs properly into account.

\section{Appendix C: Extinction map of NGC 6530}

We noted in Sect. 2 how "patchy" the density of 2MASS sources is across most of our WFI FOV (Fig. 1), and it is even more so in optical images (e.g. Paper II). We also noted that most cluster 


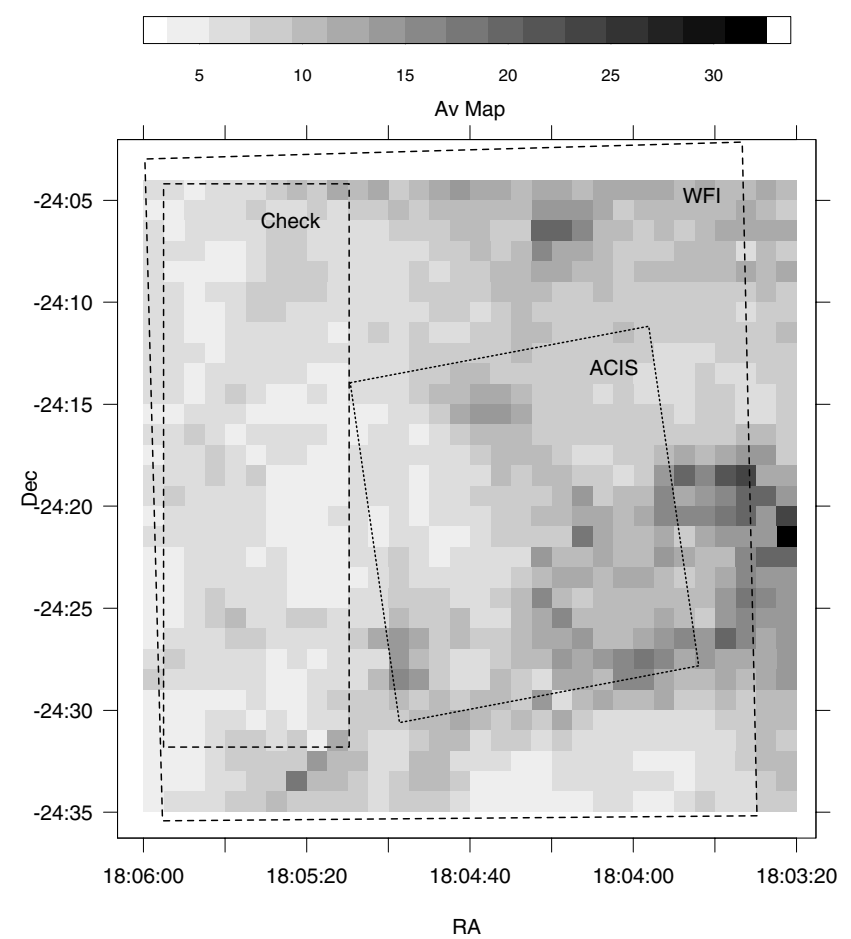

Fig. C.1. Map of optical extinction $A_{\mathrm{V}}$, derived from 2MASS colors.

stars, either selected through X-rays or IR excesses, do not appear to have very high reddening $\left(A_{\mathrm{V}} \leq 2\right)$, but most of the IRdetected field stars are very reddened, with $A_{\mathrm{V}}$ up to 20-30 mag. The most reddened stars are detected only in the IR, as expected. The 2MASS data give us the opportunity, therefore, to look through most of the cloud (perhaps except for its densest parts), and to approximately measure the total cloud optical depth (extinction). Since this is non-uniform, a proper approach is not to derive a single average extinction value, but rather to compute it towards different directions. With such a large sample of background IR sources as that made available by the 2MASS data, this spatially-resolved study can be done fruitfully.
Adopting a spatial grid with a cell size of $1.4^{\prime} \times 1^{\prime}$ over the whole WFI FOV, the number of 2MASS sources falling in each cell is in the range 10-70, with a typical value of $\sim 45$. This cell spacing can therefore be a good compromise between the need of high spatial resolution and sufficient source statistics in each cell (except for the very few cells with the largest extinction). For 2MASS sources falling in each such cell, we consider the distribution of $H-K$ colors. In order to make our procedure as insensitive as possible to the presence of cluster stars, we have excluded all X-ray sources from these samples. In any case, except perhaps in the most heavily absorbed directions, background stars are numerically dominant over cluster stars, and failing to account for (some of) these latter does not yield noticeably different results. Background stars are expected to be mostly ordinary giant stars, whose intrinsic $H-K$ colors fall in the range between $H-K \sim 0.07-0.31$ (Bessell \& Brett 1988), with a good average value being 0.2 . We then compute the median of $H-K$ for (X-ray undetected) 2MASS sources in each cell, compare it with the expected average value to obtain a reddening $E(H-K)$, and transform it to optical extinction $A_{\mathrm{V}}$ using the Rieke \& Lebofsky (1985) relations between extinction coefficients.

The resulting map of $A_{\mathrm{V}}$ for the whole WFI FOV is shown in Fig. C.1. The extinction is highly inhomogeneous inside the ACIS FOV, and reaches its highest values westward of it, near the very young object Herschel 36. A comparison with Fig. 1 (and also with Fig. 11a of Paper II) shows that high- $A_{\mathrm{V}}$ regions correspond to low source density regions, confirming that such density variations are dominated by spatially varying extinction. The Check field and the region south of the ACIS FOV are characterized by the lowest extinction values. The upper right quadrant of the ACIS FOV and its neighborhood, where a high density of IR-excess cluster stars is found, is not characterized by high extinction; on the contrary, it has a lower extinction than the regions surrounding it. 